\title{
ASYMPTOTICALLY LINEAR FRACTIONAL SCHRÖDINGER EQUATIONS
}

\author{
RAQUEL LEHRER, LILIANE A. MAIA, AND MARCO SQUASSINA
}

\begin{abstract}
By exploiting a variational technique based upon projecting over the Pohožaev manifold, we prove existence of positive solutions for a class of nonlinear fractional Schrödinger equations having a nonhomogenous nonautonomous asymptotically linear nonlinearity.
\end{abstract}

\section{Contents}

1. Introduction and main results

2. Energy levels of the limiting problem

3. Projecting on the Pohožaev manifold

4. Nonexistence results

5. Existence results

References

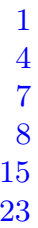

\section{INTRODUCTION AND MAIN RESULTS}

In the last few years, the study of fractional equations applied to physically relevant situations as well as to many other areas of mathematics has steadily grown. In [21,22], the authors investigate the description of anomalous diffusion via fractional dynamics and many fractional partial differential equations are derived from Lévy random walk models, extending Brownian walk models in a natural way. In particular, in [17] a fractional Schrödinger equation was obtained, which extends to a Lévy framework a classical result that path integral over Brownian trajectories leads to the standard Schrödinger equation. More precisely, let $s \in(0,1], n>2 s$ and i be the imaginary unit. Then the Schrödinger equation involving the fractional laplacian $(-\Delta)^{s}$ is

$$
\mathrm{i} \partial_{t} u=(-\Delta)^{s} u-f(x, u), \quad \text { in }(0, \infty) \times \mathbb{R}^{n},
$$

where the fractional Laplace operator is defined [10], for a suitable constant $C(n, s)$, as

$$
(-\Delta)^{s} u(x)=C(n, s) \lim _{\varepsilon \rightarrow 0^{+}} \int_{\mathbb{R}^{n} \backslash B_{\varepsilon}(x)} \frac{u(x)-u(y)}{|x-y|^{n+2 s}} d y .
$$

Though fractional Sobolev spaces are well known since the beginning of the last century, especially among harmonic analists, they have become very popular in the last few year, under the impulse of the work of Caffarelli and Silvestre [6], see again [10] and the reference within. Looking for standing wave solutions $u(t, x)=e^{\mathrm{i} \lambda t} u(x)$ of (1.1) and assuming that the nonlinearity is of the form $f(x, s)=a(x) f(s)$, we are led to study the following fractional equation

$$
(-\Delta)^{s} u+\lambda u=a(x) f(u) \text { in } \mathbb{R}^{n},
$$

for $\lambda>0$, whose variational formulation (weak solution) is

$$
\int(-\Delta)^{s / 2} u(-\Delta)^{s / 2} \varphi+\lambda \int u \varphi=\int a(x) f(u) \varphi, \quad \text { for all } \varphi \in H^{s}\left(\mathbb{R}^{n}\right) .
$$

2000 Mathematics Subject Classification. 34A08, 35Q40, 58E05.

Key words and phrases. Fractional laplacian, ground states, Pohožaev identity, critical point theory.

The first author is supported by CCET/UNIOESTE and the second author is partially supported by CNPq/ PQ 306388/2011-1. The third author is partially supported by 2009 Italian MIUR project: "Variational and Topological Methods in the Study of Nonlinear Phenomena". 
We shall assume that $f$ satisfies the following conditions:

(f1) $f \in C^{1}\left(\mathbb{R}, \mathbb{R}^{+}\right), \quad f(s)=0$ for $s \leq 0, \quad \lim _{s \rightarrow 0^{+}} \frac{f(s)}{s}=0$;

(f2) $\lim _{s \rightarrow+\infty} \frac{f(s)}{s}=1$;

(f3) if $F(s):=\int_{0}^{s} f(t) d t$ and $Q(s):=\frac{1}{2} f(s) s-F(s)$, then there exists $D \geq 1$ such that $Q(s) \leq D Q(t), \quad$ for all $s \in[0, t], \quad \lim _{s \rightarrow+\infty} Q(s)=+\infty$.

On the function $a: \mathbb{R}^{n} \rightarrow \mathbb{R}$, we will assume the following conditions:

(A1) $a \in C^{2}\left(\mathbb{R}^{n}, \mathbb{R}^{+}\right), \quad \inf _{\mathbb{R}^{n}} a>0$;

(A2) $\lim _{|x| \rightarrow+\infty} a(x)=a_{\infty}>\lambda$;

(A3) $\nabla a(x) \cdot x \geq 0$, for all $x \in \mathbb{R}^{n}$, with strict inequality on a set of positive measure;

(A4) $a(x)+\frac{\nabla a(x) \cdot x}{n}<a_{\infty}$, for all $x \in \mathbb{R}^{n}$;

(A5) $\nabla a(x) \cdot x+\frac{x \cdot \mathcal{H}_{a}(x) \cdot x}{n} \geq 0$, for all $x \in \mathbb{R}^{n}$, being $\mathcal{H}_{a}$ the Hessian matrix of $a$.

Now we can state our main results. Consider the energy functional $I: H^{s}\left(\mathbb{R}^{n}\right) \rightarrow \mathbb{R}$,

$$
I(u):=\frac{1}{2} \int\left|(-\Delta)^{s / 2} u\right|^{2}+\frac{\lambda}{2} \int u^{2}-\int a(x) F(u),
$$

naturally associated with equation (1.2). Then, we have the following nonexistence result

Theorem 1.1. Assume that $(A 1)-(A 5)$ and $(f 1)-(f 3)$ hold and consider

$$
\mathcal{P}:=\left\{u \in H^{s}\left(\mathbb{R}^{n}\right) \backslash\{0\}: \frac{n-2 s}{2} \int\left|(-\Delta)^{s / 2} u\right|^{2}=n \int\left(\left(a(x)+\frac{\nabla a(x) \cdot x}{n}\right) F(u)-\frac{\lambda}{2} u^{2}\right)\right\} .
$$

Then, the infimum

$$
\inf _{u \in \mathcal{P}} I(u)
$$

is not a critical level of $I$ and the infimum is not achieved.

Consider now also the limiting problem

$$
(-\Delta)^{s} u+\lambda u=a_{\infty} f(u) \text { in } \mathbb{R}^{n} .
$$

We shall denote by $I_{\infty}: H^{s}\left(\mathbb{R}^{n}\right) \rightarrow \mathbb{R}$,

$$
I_{\infty}(u):=\frac{1}{2} \int\left|(-\Delta)^{s / 2} u\right|^{2}+\frac{\lambda}{2} \int u^{2}-\int a_{\infty} F(u),
$$

its associated energy functional. In Section 2 we shall discuss some properties of least energy critical values of this functional. In passing, we observe that by combining the results of [20] (see e.g. Theorem 4.1 therein) with an adaptation of [5, (i) of Lemma 1] to the fractional framework, it is possible to prove that any least energy solution to (1.5) is radially symmetric and decreasing and of fixed sign.

We have the following existence result

Theorem 1.2. Assume that $(A 1)-(A 5),(f 1)-(f 3)$ hold and that the following facts hold

(1) $f \in C^{1}(\mathbb{R}) \cap \operatorname{Lip}\left(\mathbb{R}, \mathbb{R}^{+}\right)$and there exists $\tau>0$ such that $\lim _{s \rightarrow 0^{+}} \frac{f^{\prime}(s)}{s^{\tau}}=0$;

(2) $\left\|a_{\infty}-a\right\|_{L^{\infty}}$ is sufficiently small; 
(3) the least energy level $c_{\infty}$ of (1.5) is an isolated radial critical level for $I_{\infty}$ or equation (1.5) admits a unique positive solution which is radially symmetric about some point.

Then the nonautonomous problem

$$
(-\Delta)^{s} u+\lambda u=a(x) f(u) \quad \text { in } \mathbb{R}^{n},
$$

admits a nontrivial nonnegative solution $u \in H^{s}\left(\mathbb{R}^{n}\right)$.

These results extend the corresponding results in [18] to the fractional case. The framework employed and ideas of the proofs of our main results follow closely those found in [18]. However, the nonlocal character of the fractional laplacian requires to overcome several additional difficulties.

Theorem 1.2 follows under uniqueness of positive radial solutions of (1.5) or isolatedness assumption on the least energy level of $I_{\infty}$. To our knowledge, in the case $s \in(0,1)$, the isolatedness or uniqueness assumption of Theorem 1.2 are unknown in the current literature. In the case $s=1$, it follows for instance by the uniqueness result by Serrin-Tang [24], under suitable assumptions of $f(s)$ for large values of $s$, which are compatible with the model nonlinearity

$$
f(s)=\frac{s^{3}}{1+s^{2}}, \quad \text { for } s \geq 0, \quad f(s)=0, \quad \text { for } s \leq 0 .
$$

In fact, assumptions (H1)-(H2) in [24, Theorem 1] are fulfilled with $b=\left(\lambda /\left(a_{\infty}-\lambda\right)\right)^{1 / 2}>0$, where $a_{\infty}>\lambda$. For the case of superquadratic nonlinearities $f(s)=s^{p}$, nondegeneracy and uniqueness properties of ground state solutions of (1.5) where recently proved in [13,14], so assumption (3) of Theorem 1.2 is expected to be fulfilled. Semi-linear Schrödinger equations associated with the asymptotically linear model nonlinearity (1.6) are one of the main motivations for developing the technique in this paper. For the physical background in the local case $s=1$, see $[27,28]$.

In [7], the author considers asymptotically linear fractional NLS with an external potential $V$ which provides compactness directly via coercivity. We also refer the reader to the contributions $[8,12]$ where the case of a superquadratic nonlinearity is covered for the fractional laplacian obtaining existence, regularity and qualitative properties of solutions. In the superquadratic case, as known, one can also exploit the Nehari manifold associated with the problem. On the other hand, when the nonlinear term is nonhomogeneous and asymptotically linear, as it was pointed out by Costa and Tehrani in [9], in general, not every nonzero function can be projected onto the Nehari manifold or it may happen that the projection is not uniquely determined. In turn, as exploitied in other contributions $[3,16,18]$, we shall look at projections onto the Pohožaev manifold in place of the Nehari constraint in order to prove Theorem 1.1 and 1.2.

A few additional remarks. Conditions $(A 2),(A 3)$ and $(A 4)$ imply

$$
\nabla a(x) \cdot x \rightarrow 0, \quad \text { if } \quad|x| \rightarrow+\infty,
$$

while $(f 1)$ and $(f 2)$ imply that, given $\varepsilon>0$ and $2<p \leq 2 n /(n-2 s)$, there exists $C_{\varepsilon}>0$ with

$$
|F(s)| \leq \frac{\varepsilon}{2}|s|^{2}+C_{\varepsilon}|s|^{p}, \quad \text { for all } s \in \mathbb{R} .
$$

In what follows we will denote

$$
\|u\|_{H^{s}}=\left(\int\left|(-\Delta)^{s / 2} u\right|^{2}+\int \lambda u^{2}\right)^{1 / 2},
$$

as the norm in $H^{s}\left(\mathbb{R}^{n}\right)$, which is equivalent to the standard norm of $H^{s}\left(\mathbb{R}^{n}\right)$. We will also denote by $\|u\|_{p}$ de usual norm of $L^{p}\left(\mathbb{R}^{n}\right)$. We define $I: H^{s}\left(\mathbb{R}^{n}\right) \rightarrow \mathbb{R}$ as the functional associated with $(1.2)$

$$
I(u):=\frac{1}{2} \int\left|(-\Delta)^{s / 2} u\right|^{2}-\int G(x, u), \quad G(x, u):=a(x) F(u)-\frac{\lambda}{2} u^{2} .
$$


Since $f(s)=0$ on $\mathbb{R}^{-}$, it follows that any weak solution $u \in H^{s}\left(\mathbb{R}^{n}\right)$ for $(1.2)$ is nonnegative. In fact, by choosing $\varphi=u^{-} \in H^{s}\left(\mathbb{R}^{n}\right)$ in the variational formulation (1.3) yields

$$
\int(-\Delta)^{s / 2} u(-\Delta)^{s / 2} u^{-}=\int a(x) f(u) u^{-}-\lambda \int u u^{-}=\lambda \int\left(u^{-}\right)^{2} .
$$

Hence, if $C(n, s)$ is the normalization constant in the definition of $(-\Delta)^{s}$, we obtain

$$
\begin{aligned}
& \int(-\Delta)^{s / 2} u(-\Delta)^{s / 2} u^{-}=\int u^{-}(-\Delta)^{s} u^{+}-\left\|(-\Delta)^{s / 2} u^{-}\right\|_{2}^{2} \\
& =\frac{C(n, s)}{2} \iint \frac{\left(u^{+}(x)-u^{+}(y)\right)\left(u^{-}(x)-u^{-}(y)\right)}{|x-y|^{n+2 s}} d x d y-\left\|(-\Delta)^{s / 2} u^{-}\right\|_{2}^{2} \\
& =-C(n, s) \iint \frac{u^{+}(x) u^{-}(y)}{|x-y|^{n+2 s}} d x d y-\left\|(-\Delta)^{s / 2} u^{-}\right\|_{2}^{2} \leq-\left\|(-\Delta)^{s / 2} u^{-}\right\|_{2}^{2} .
\end{aligned}
$$

In turn we get $\left\|u^{-}\right\|_{H^{s}}^{2}=\left\|u^{-}\right\|_{2}^{2}+\left\|(-\Delta)^{s / 2} u^{-}\right\|_{2}^{2}=0$, namely $u^{-}=0$, hence the assertion.

\section{ENERGy LEVElS OF THE LIMiting PROBLEM}

In this section we study the following equation for $s \in(0,1)$ and $n>2 s$,

$$
(-\Delta)^{s} u+\lambda u=a_{\infty} f(u), \quad \text { in } \mathbb{R}^{n},
$$

where $\lambda>0$ and $a_{\infty}>\lambda$. We shall assume that $F$ satisfies the growth estimate (1.8). Our aim is to provide a Mountain Pass characterization for least energy solutions which is the counterpart of the main result of [16]. Let the Hilbert space $H^{s}\left(\mathbb{R}^{n}\right)$ be endowed with the norm (1.9) and let $I_{\infty}: H^{s}\left(\mathbb{R}^{n}\right) \rightarrow \mathbb{R}$ be the functional corresponding to (2.1), namely

$$
I_{\infty}(u)=\frac{1}{2} \int\left|(-\Delta)^{s / 2} u\right|^{2}-\int G_{\infty}(u)
$$

where we have set

$$
G_{\infty}(u)=\int_{0}^{u} g_{\infty}(t) d t=\int_{0}^{u}\left(a_{\infty} f(t)-\lambda t\right) d t=a_{\infty} F(u)-\frac{\lambda}{2} u^{2} .
$$

We say that a solution $u$ of (2.1) is a least energy solution to (2.1) if

$$
I_{\infty}(u)=m, \quad m:=\inf \left\{I_{\infty}(u): u \in H^{s}\left(\mathbb{R}^{n}\right) \backslash\{0\} \text { is a solution of }(2.1)\right\} .
$$

As stated in [23, Theorem 1.1], the Pohožaev identity associated with (2.1) is given by

$$
(n-2 s) \int u g_{\infty}(u)=2 n \int G_{\infty}(u),
$$

where $g_{\infty}$ and $G_{\infty}$ are defined as before. Also, if $u$ and $v$ belong to $H^{s}\left(\mathbb{R}^{n}\right)$, then

$$
\int v(-\Delta)^{s} u=\int(-\Delta)^{s / 2} u(-\Delta)^{s / 2} v
$$

which yields in turn

$$
\int u g_{\infty}(u)=\int\left|(-\Delta)^{s / 2} u\right|^{2}=\frac{C(n, s)}{2}[u]_{H^{s}}^{2}, \quad[u]_{H^{s}}:=\left(\iint \frac{|u(x)-u(y)|^{2}}{|x-y|^{n+2 s}} d x d y\right)^{1 / 2} .
$$

Therefore, the Pohožaev identity may be written as (see also [8, Proposition 4.1] for a different proof)

$$
(n-2 s) \int\left|(-\Delta)^{s / 2} u\right|^{2}=2 n \int G_{\infty}(u) .
$$

For the following, it is convenient to introduce the set

$$
\mathcal{P}_{\infty}:=\left\{u \in H^{s}\left(\mathbb{R}^{n}\right) \backslash\{0\}: u \text { satisfies identity }(2.2)\right\} .
$$


We also consider we set of paths

$$
\Gamma_{\infty}:=\left\{\gamma \in C\left([0,1], H^{s}\left(\mathbb{R}^{n}\right)\right): \gamma(0)=0, I_{\infty}(\gamma(1))<0\right\},
$$

and define the min-max Mountain Pass level (see [2])

$$
c_{\infty}:=\min _{\gamma \in \Gamma_{\infty}} \max _{t \in[0,1]} I_{\infty}(\gamma(t)) .
$$

The main result of the section is the following

Theorem 2.1. $c_{\infty}=m$.

In order to prove the result we need the following Lemmas.

Lemma 2.2. Let $w \in H^{s}\left(\mathbb{R}^{n}\right)$ be a least energy solution to (2.1). Then there exists $\gamma \in \Gamma_{\infty}$ such that

$$
w \in \gamma([0,1]), \quad \max _{t \in[0,1]} I_{\infty}(\gamma(t))=I_{\infty}(w)=m .
$$

Proof. Consider a least energy solution $w$ of (2.1), which exists e.g. by [8, Theorem 1.1]. Then we can define the continuous path $\alpha:[0, \infty) \rightarrow H^{s}\left(\mathbb{R}^{n}\right)$ by setting $\alpha(t)(x):=w(x / t)$, if $t>0$, and $\alpha(0):=0$. Then, by construction, we have $I_{\infty}(\alpha(0))=0$ and

$$
\begin{aligned}
I_{\infty}(\alpha(t)) & =\frac{1}{2} \int\left|(-\Delta)^{s / 2} w(x / t)\right|^{2}-\int G_{\infty}(w(x / t)) \\
& =\frac{t^{n-2 s}}{2} \int\left|(-\Delta)^{s / 2} w(x)\right|^{2}-t^{n} \int G_{\infty}(w), \quad t>0 .
\end{aligned}
$$

Then, taking the derivative, we obtain

$$
\begin{aligned}
\frac{d}{d t} I_{\infty}(\alpha(t)) & =\frac{(n-2 s)}{2} t^{n-2 s-1} \int\left|(-\Delta)^{s / 2} w\right|^{2}-n t^{n-1} \int G_{\infty}(w) \\
& =\frac{t^{n-2 s-1}}{2}\left\{(n-2 s) \int\left|(-\Delta)^{s / 2} w\right|^{2}-2 n t^{2 s} \int G_{\infty}(w)\right\}
\end{aligned}
$$

Since $w$ is a solution of (2.1), it satisfies the Pohožaev identity (2.2), therefore

$$
\frac{d}{d t} I_{\infty}(\alpha(t))=\frac{t^{n-2 s-1}}{2}(n-2 s)\left(1-t^{2 s}\right) \int\left|(-\Delta)^{s / 2} w\right|^{2} .
$$

Then, since $n>2 s$, the map $\left\{t \mapsto I_{\infty}(\alpha(t))\right\}$ achieves the maximum value at $t=1$. By choosing $L>0$ sufficiently large and recalling $(2.2)$ again to guarantee $\int G_{\infty}(w)>0$, we have

$$
\max _{0 \leq t \leq L} I_{\infty}(\alpha(t))=I_{\infty}(\alpha(1))=I_{\infty}(w)=m, \quad I_{\infty}(\alpha(L))<0 .
$$

Taking $\gamma(t):=\alpha(t L)$, we have that $\gamma \in \Gamma_{\infty}$ and the result follows.

Lemma 2.3. $\gamma([0,1]) \cap \mathcal{P}_{\infty} \neq \emptyset$, for all $\gamma \in \Gamma_{\infty}$.

Proof. Consider the functional associated with the Pohožaev identity (2.2),

$$
J_{\infty}(u):=\frac{n-2 s}{2} \int\left|(-\Delta)^{s / 2} u\right|^{2}-n \int G_{\infty}(u), \quad u \in H^{s}\left(\mathbb{R}^{n}\right) .
$$

We will first prove that there exists $\rho>0$ such that, if $0<\|u\|_{H^{s}} \leq \rho$, then $J(u)>0$. We have

$$
\begin{aligned}
J_{\infty}(u) & =\frac{n-2 s}{2} \int\left|(-\Delta)^{s / 2} u\right|^{2}-n a_{\infty} \int F(u)+\frac{n \lambda}{2} \int u^{2} \\
& \geq \frac{n-2 s}{2}\|u\|_{H^{s}}^{2}-n a_{\infty} \int F(u) .
\end{aligned}
$$


Then, by virtue of (1.8) and the fractional Sobolev inequality [10, Theorem 6.7], we obtain

$$
\begin{aligned}
J_{\infty}(u) & \geq \frac{n-2 s}{2}\|u\|_{H^{s}}^{2}-\frac{n \varepsilon a_{\infty}}{2 \lambda} \int \lambda u^{2}-n a_{\infty} C_{\varepsilon} \int|u|^{p} \\
& \geq \frac{1}{2}\left(n-2 s-\frac{n \varepsilon a_{\infty}}{\lambda}\right)\|u\|_{H^{s}}^{2}-n a_{\infty} C_{\varepsilon}\|u\|_{H^{s}}^{p} .
\end{aligned}
$$

Take now $\varepsilon>0$ so small that $n-2 s-n \varepsilon a_{\infty} / \lambda>0$ and then choose $\rho>0$ small enough so that $J_{\infty}(u)>0$ if $0<\|u\|_{H^{s}} \leq \rho$, which is possible, since $p>2$. Observe now that

$$
J_{\infty}(u)=n I_{\infty}(u)-s \int\left|(-\Delta)^{s / 2} u\right|^{2}
$$

If $\gamma \in \Gamma_{\infty}$, we have $J_{\infty}(\gamma(0))=0$ and $J_{\infty}(\gamma(1)) \leq n I_{\infty}(\gamma(1))<0$. Then, by continuity, there exists $\sigma \in(0,1)$ such that $\|\gamma(\sigma)\|_{H^{s}} \geq \rho$ and $J_{\infty}(\gamma(\sigma))=0$. This means $\gamma(\sigma) \in \mathcal{P}_{\infty}$, concluding the proof.

Lemma 2.4. We have

$$
m=\inf _{u \in \mathcal{P}_{\infty}} I_{\infty}(u)
$$

Proof. If we set

$$
\mathcal{S}_{\infty}=\left\{u \in H^{s}\left(\mathbb{R}^{n}\right): \int G_{\infty}(u)=1\right\}
$$

it follows that $\Phi: \mathcal{S}_{\infty} \rightarrow \mathcal{P}_{\infty}$ defined by

$$
\Phi(u)(x):=u\left(\frac{x}{t_{u}}\right), \quad t_{u}:=\left(\frac{n-2 s}{2 n}\right)^{1 / 2 s}\left\|(-\Delta)^{s / 2} u\right\|_{2}^{1 / s}
$$

establishes a bijective correspondence and

$$
I_{\infty}(\Phi(u))=\frac{s}{n}\left(\frac{n-2 s}{2 n}\right)^{(n-2 s) / 2 s}\left\|(-\Delta)^{s / 2} u\right\|_{2}^{n / s}, \quad u \in \mathcal{S}_{\infty}
$$

yielding in turn

$$
\inf _{u \in \mathcal{P}_{\infty}} I_{\infty}(u)=\inf _{u \in \mathcal{S}_{\infty}} I_{\infty}(\Phi(u))=\inf _{u \in \mathcal{S}_{\infty}} \frac{s}{n}\left(\frac{n-2 s}{2 n}\right)^{(n-2 s) / 2 s}\left\|(-\Delta)^{s / 2} u\right\|_{2}^{n / s}=m,
$$

since the last infimum is achieved and the corresponding value equals the least energy level $m$. This can be proved by performing calculations similar to those of [5, proof of (i) of Lemma 1].

Proof of Theorem 2.1 concluded. By combining Lemma 2.3 with 2.4 we immediately obtain $m \leq c_{\infty}$. Considering the path $\gamma \in \Gamma_{\infty}$ provided by Lemma 2.3, we have

$$
\max _{0 \leq t \leq 1} I_{\infty}(\gamma(t))=I_{\infty}(w)=m
$$

By taking the infimum over $\Gamma_{\infty}$ yields

$$
\inf _{\gamma \in \Gamma_{\infty}} \max _{t \in[0,1]} I_{\infty}(\gamma(t)) \leq m,
$$

so that $c_{\infty} \leq m$, which concludes the proof. 


\section{Projecting on the PohožAev manifold}

From [23, Proposition 1.12], if $u \in H^{s}\left(\mathbb{R}^{n}\right)$ is a solution of (1.2), then $u$ satisfies de Pohožaev identity

$$
\frac{n-2 s}{2} \int\left|(-\Delta)^{s / 2} u\right|^{2}=n \int\left(\left(a(x)+\frac{\nabla a(x) \cdot x}{n}\right) F(u)-\frac{\lambda}{2} u^{2}\right) .
$$

Furthermore, we define the Pohožaev manifold associated with (1.2) by

$$
\mathcal{P}:=\left\{u \in H^{s}\left(\mathbb{R}^{n}\right) \backslash\{0\}: u \text { satisfies identity }(3.1)\right\}
$$

We first have the following

Lemma 3.1. Let the functional $J: H^{s}\left(\mathbb{R}^{n}\right) \rightarrow \mathbb{R}$ be defined by

$$
J(u):=\frac{n-2 s}{2} \int\left|(-\Delta)^{s / 2} u\right|^{2}-n \int\left(\left(a(x)+\frac{\nabla a(x) \cdot x}{n}\right) F(u)-\frac{\lambda u^{2}}{2}\right) .
$$

Then, it holds that

a) $\{u \equiv 0\}$ is an isolated point of $J^{-1}(\{0\})$;

b) $\mathcal{P}:=\left\{u \in H^{s}\left(\mathbb{R}^{n}\right) \backslash\{0\}: J(u)=0\right\}$ is a closed set.

c) $\mathcal{P}$ is a $C^{1}$ manifold.

d) There exists $\sigma>0$ such that $\|u\|_{H^{s}}>\sigma$, for all $u \in \mathcal{P}$.

Proof. (a) Using condition (A4), we get

$$
\begin{aligned}
J(u) & =\frac{n-2 s}{2} \int\left|(-\Delta)^{s / 2} u\right|^{2}-n \int\left(\left(a(x)+\frac{\nabla a(x) \cdot x}{n}\right) F(u)-\frac{\lambda u^{2}}{2}\right) \\
& >\frac{n-2 s}{2} \int\left|(-\Delta)^{s / 2} u\right|^{2}-n \int\left(a_{\infty} F(u)-\lambda \frac{u^{2}}{2}\right) \\
& \geq \frac{n-2 s}{2}\|u\|_{H^{s}}^{2}-n a_{\infty} \int F(u) .
\end{aligned}
$$

By virtue of the fractional Sobolev embedding [10, Theorem 6.7] and (1.8), we obtain

$$
\begin{aligned}
J(u) & \geq \frac{n-2 s}{2}\|u\|_{H^{s}}^{2}-\frac{n \varepsilon a_{\infty}}{2 \lambda} \int \lambda u^{2}-n a_{\infty} C_{\varepsilon} \int|u|^{p} \\
& \geq \frac{1}{2}\left(n-2 s-\frac{n \varepsilon a_{\infty}}{\lambda}\right)\|u\|_{H^{s}}^{2}-n a_{\infty} C_{\varepsilon}\|u\|_{H^{s}}^{p} .
\end{aligned}
$$

Take $\varepsilon>0$ with $n-2 s-n \varepsilon a_{\infty} / \lambda>0$. For $\rho>0$ small enough, $J(u)>0$ if $0<\|u\|_{H^{s}}<\rho$.

(b) $J(u)$ is a $C^{1}$ functional, thus $\mathcal{P} \cup\{0\}=J^{-1}(\{0\})$ is a closed subset. Moreover, $\{u \equiv 0\}$ is an isolated point in $J^{-1}(\{0\})$ and the assertion follows.

(c) Considering the derivative of $J$ at $u$ and applied at $u$ yields

$$
J^{\prime}(u)(u)=(n-2 s) \int\left|(-\Delta)^{s / 2} u\right|^{2}-n \int\left(a(x) f(u) u-\lambda u^{2}\right)-\int \nabla a(x) \cdot x f(u) u .
$$


Since $u \in \mathcal{P}$, it follows that $u$ satisfies (3.1) and, using formula (3.1) into (3.2), we obtain

$$
\begin{aligned}
J^{\prime}(u)(u) & =2 n \int a(x) F(u)-n \lambda \int u^{2}+2 \int \nabla a(x) \cdot x F(u) \\
& -n \int a(x) f(u) u+n \lambda \int u^{2}-\int \nabla a(x) \cdot x f(u) u \\
& =2 n \int\left(a(x)+\frac{\nabla a(x) \cdot x}{n}\right) F(u) \\
& -n \int\left(a(x)+\frac{\nabla a(x) \cdot x}{n}\right) f(u) u \\
& =2 n \int\left(a(x)+\frac{\nabla a(x) \cdot x}{n}\right)\left(F(u)-\frac{1}{2} f(u) u\right)<0
\end{aligned}
$$

in light of $(A 1),(A 3)$ and $(f 3)$. Then, if $u \in \mathcal{P}$, then $J^{\prime}(u)(u)<0$. This shows that the set $\mathcal{P}$ is a $C^{1}$ manifold.

(d) Since 0 is isolated in $J^{-1}(\{0\})$, there is a ball $\|u\|_{H^{s}} \leq \sigma$ which does not intersect $\mathcal{P}$.

\section{Nonexistence Results}

In this section we get relations between the Pohožaev manifold $\mathcal{P}$ associated with (1.2) and the Pohožaev manifold $\mathcal{P}_{\infty}$ for the limiting problem (2.1). Recall that

$$
\mathcal{P}_{\infty}=\left\{u \in H^{s}\left(\mathbb{R}^{n}\right) \backslash\{0\}: J_{\infty}(u)=0\right\},
$$

where $J_{\infty}$ is defined as in (2.4). Notice that the hypotheses $(A 3)-(A 4)$ imply that $I_{\infty}(u)<I(u)$ for every $u$ in $H^{s}\left(\mathbb{R}^{n}\right) \backslash\{0\}$. If $p$ is defined as in (1.4), we will show in this section that $p=c_{\infty}$, that this level is not critical for $I$ and in turn that it is not achieved.

Lemma 4.1. If $\int G_{\infty}(u)>0$, there exist unique $\vartheta_{1}, \vartheta_{2}>0$ with $u\left(\cdot / \vartheta_{1}\right) \in \mathcal{P}_{\infty}$ and $u\left(\cdot / \vartheta_{2}\right) \in \mathcal{P}$.

Proof. First, we consider the case of $\mathcal{P}_{\infty}$. Consider the function $\varphi:(0, \infty) \rightarrow \mathbb{R}$ defined by

$$
\varphi(\vartheta):=I_{\infty}(u(x / \vartheta))=\frac{\vartheta^{n-2 s}}{2} \int\left|(-\Delta)^{s / 2} u\right|^{2}-\vartheta^{n} \int G_{\infty}(u)
$$

Taking the derivative of $\varphi$, we obtain

$$
\begin{aligned}
\varphi^{\prime}(\vartheta) & =\frac{\vartheta^{n-2 s-1}}{2}\left((n-2 s) \int\left|(-\Delta)^{s / 2} u\right|^{2}-2 n \vartheta^{2 s} \int G_{\infty}(u)\right) \\
& =\frac{1}{\vartheta}\left(\frac{n-2 s}{2} \int\left|(-\Delta)^{s / 2} u(x / \vartheta)\right|^{2}-n \int G_{\infty}(u(x / \vartheta))\right)=\frac{J_{\infty}(u(\cdot / \vartheta))}{\vartheta} .
\end{aligned}
$$

Then, $\varphi^{\prime}(\vartheta)=0$ if and only if either $\vartheta=0$ or

$$
\vartheta=\vartheta_{1}=\left(\frac{n-2 s}{2 n} \frac{\int\left|(-\Delta)^{s / 2} u\right|^{2}}{\int G_{\infty}(u)}\right)^{1 / 2 s}>0 .
$$

Since by the formula for $\varphi^{\prime}$ we have $u(x / \vartheta) \in \mathcal{P}_{\infty}$ if and only if $\varphi^{\prime}(\vartheta)=0$ for some $\vartheta>0$, we have the result. In passing, we observe that $\varphi$ is positive for $\vartheta>0$ small while it is negative for $\vartheta>0$ large, so that the unique critical point of $\varphi$ corresponds to a global maximum point for $\varphi$. Now we 
turn to the case of $\mathcal{P}$. First, we define the function $\Psi:(0, \infty) \rightarrow \mathbb{R}$ by

$$
\begin{aligned}
\Psi(\vartheta) & :=I(u(x / \vartheta))=\frac{\vartheta^{n-2 s}}{2} \int\left|(-\Delta)^{s / 2} u\right|^{2}-\int G(x, u(x / \vartheta)) \\
& =\frac{\vartheta^{n-2 s}}{2} \int\left|(-\Delta)^{s / 2} u\right|^{2}-\int\left(a(x) F(u(x / \vartheta))-\lambda \frac{u^{2}(x / \vartheta)}{2}\right) \\
& =\frac{\vartheta^{n-2 s}}{2} \int\left|(-\Delta)^{s / 2} u\right|^{2}-\vartheta^{n} \int\left(a(\vartheta x) F(u)-\lambda \frac{u^{2}}{2}\right) .
\end{aligned}
$$

Taking the derivative of $\Psi$ and recalling that $n>2 s$, we obtain:

$$
\begin{aligned}
\Psi^{\prime}(\vartheta) & =\frac{n-2 s}{2} \vartheta^{n-2 s-1} \int\left|(-\Delta)^{s / 2} u\right|^{2}-n \vartheta^{n-1} \int\left(a(\vartheta x) F(u)-\lambda \frac{u^{2}}{2}\right) \\
& -\vartheta^{n} \int \nabla a(\vartheta x) \cdot x F(u) \\
& =\vartheta^{n-2 s-1}\left\{\frac{n-2 s}{2} \int\left|(-\Delta)^{s / 2} u\right|^{2}-n \vartheta^{2 s} \int\left(a(\vartheta x) F(u)-\lambda \frac{u^{2}}{2}\right)\right. \\
& \left.-\vartheta^{2 s} \int \nabla a(\vartheta x) \cdot(\vartheta x) F(u)\right\} \\
& =\frac{1}{\vartheta}\left\{\frac{n-2 s}{2} \int\left|(-\Delta)^{s / 2} u(x / \vartheta)\right|^{2}-n \int\left(a(x) F(u(x / \vartheta))-\lambda \frac{u^{2}(x / \vartheta)}{2}\right)\right. \\
& \left.-\int \nabla a(x) \cdot x F(u(x / \vartheta))\right\}=\frac{J(u(\cdot / \vartheta))}{\vartheta} .
\end{aligned}
$$

Hence, $u(\cdot / \vartheta) \in \mathcal{P}$ if and only if $\Psi^{\prime}(\vartheta)=0$, for some $\vartheta>0$. Notice that, in view of $(A 2)$ and $(1.7)$ and the Lebesgue Dominated Convergence Theorem, we get

$$
\begin{aligned}
& \lim _{\vartheta \rightarrow \infty} \int a(\vartheta x) F(u)-\lambda \frac{u^{2}}{2}=\int G_{\infty}(u), \\
& \lim _{\vartheta \rightarrow \infty} \int \nabla a(\vartheta x) \cdot(\vartheta x) F(u)=0 .
\end{aligned}
$$

Therefore, if $\vartheta>0$ is sufficiently large, then

$$
\Psi^{\prime}(\vartheta)=\vartheta^{n-2 s-1}\left\{\frac{n-2 s}{2} \int\left|(-\Delta)^{s / 2} u\right|^{2}-n \vartheta^{2 s}\left(\int G_{\infty}(u)+o_{\vartheta}(1)\right)\right\} .
$$

Since $\int G_{\infty}(u)>0$, it follows that $\Psi^{\prime}(\vartheta)<0$, for $\vartheta>0$ sufficiently large. On the other hand, if $\vartheta>0$ is sufficiently small we have that condition $(A 4)$, together with $(A 1)-(A 3)$ yield

$$
\begin{gathered}
0<a(x)+\frac{\nabla a(x) \cdot x}{n}<a_{\infty}, \\
-\frac{\lambda}{2} \int u^{2} \leq \int\left(\left(a(\vartheta x)+\frac{\nabla a(\vartheta x) \cdot(\vartheta x)}{n}\right) F(u)-\lambda \frac{u^{2}}{2}\right)<\int G_{\infty}(u) \leq \frac{a_{\infty} C}{2} \int u^{2},
\end{gathered}
$$

where $C$ is a positive constant independent of $\vartheta$. Thus, taking $\vartheta>0$ sufficiently small in $\Psi^{\prime}(\vartheta)$, we obtain $\Psi^{\prime}(\vartheta)>0$. Since $\Psi^{\prime}$ is continuous, there exists $\vartheta_{2}=\vartheta_{2}(u)>0$ such that $\Psi^{\prime}\left(\vartheta_{2}\right)=0$, which means that $u\left(\cdot / \vartheta_{2}\right) \in \mathcal{P}$. To show the uniqueness of $\vartheta_{2}$, note that $\Psi^{\prime}(\vartheta)=0$ implies

$$
\frac{n-2 s}{2} \int\left|(-\Delta)^{s / 2} u\right|^{2}=n \vartheta^{2 s} h(\vartheta), \quad h(\vartheta):=\int\left(\left(a(\vartheta x)+\frac{\nabla a(\vartheta x) \cdot(\vartheta x)}{n}\right) F(u)-\frac{\lambda u^{2}}{2}\right)
$$

with $\vartheta>0$. Taking the derivative of $h$ we end up with

$$
h^{\prime}(\vartheta)=\frac{1}{\vartheta} \int\left(\nabla a(\vartheta x) \cdot(\vartheta x)+\frac{(\vartheta x) \cdot \mathcal{H}_{a}(\vartheta x) \cdot(\vartheta x)}{n}+\frac{\nabla a(\vartheta x) \cdot(\vartheta x)}{n}\right) F(u) .
$$


Hypotheses $(A 3)$ and $(A 5)$ imply that $h^{\prime}(\vartheta)>0$. Therefore, $h$ is an increasing function of $\vartheta$ and hence there exists a unique $\vartheta>0$ such that the identity in (4.3) holds. As for the functional $\varphi$, the above arguments show that $\Psi$ is positive for $\vartheta>0$ small while it is negative for $\vartheta>0$ large, and hence the unique critical point of $\Psi$ corresponds to a global maximum point for $\Psi$.

Consider the open subset of $H^{s}\left(\mathbb{R}^{n}\right)$

$$
\mathcal{O}=\left\{u \in H^{s}\left(\mathbb{R}^{n}\right) \backslash\{0\}: \int G_{\infty}(u)>0\right\} .
$$

Then we have the following

Lemma 4.2. The map defined by $\mathcal{O} \ni u \mapsto \theta_{2}(u) \in(0, \infty)$, such that $u\left(\cdot / \theta_{2}(u)\right) \in \mathcal{P}$, is continuous.

Proof. Let $u \in \mathcal{O}$ and consider $\left(u_{j}\right) \subset \mathcal{O}$ such that $u_{j} \rightarrow u$ in $H^{s}\left(\mathbb{R}^{n}\right)$ as $j \rightarrow \infty$. First note that $\vartheta_{2}\left(u_{j}\right)$ is bounded. Indeed, consider the expression $(4.3)$ of $\psi^{\prime}=0$ in the proof of Lemma 4.1 for $u_{j}$ and $\vartheta_{2}\left(u_{j}\right)$

$$
\frac{n-2 s}{2} \int\left|(-\Delta)^{s / 2} u_{j}\right|^{2}=n \vartheta_{2}^{2 s}\left(u_{j}\right) \int\left(\left(a\left(\vartheta_{2}\left(u_{j}\right) x\right)+\frac{\nabla a\left(\vartheta_{2}\left(u_{j}\right) x\right) \cdot\left(\vartheta_{2}\left(u_{j}\right) x\right)}{n}\right) F\left(u_{j}\right)-\frac{\lambda u_{j}^{2}}{2}\right) .
$$

Suppose by contradiction that $\vartheta_{2}\left(u_{j}\right) \rightarrow \infty$ as $j \rightarrow \infty$, along a suitable subsequence. Then, in light of the assumptions on $a$ and $F$ and by Lebesgue Dominated Convergence Theorem, the right-hand side of the above equation goes to infinity while the left-hand converges to $(n-2 s) / 2\left\|(-\Delta)^{s / 2} u\right\|_{2}^{2}$, which is a contradiction. Hence, $\vartheta_{2}\left(u_{j}\right)$ admits a convergent subsequence, say $\vartheta_{2}\left(u_{j}\right) \rightarrow \bar{\vartheta}$ as $j \rightarrow \infty$. In turn, by Lebesgue Dominated Convergence Theorem, as $j \rightarrow \infty$, we have

$$
\begin{aligned}
\int a\left(\vartheta_{2}\left(u_{j}\right) x\right) F\left(u_{j}\right) & \rightarrow \int a(\bar{\vartheta} x) F(u), \\
\int \nabla a\left(\vartheta_{2}\left(u_{j}\right) x\right) \cdot\left(\vartheta_{2}\left(u_{j}\right) x\right) F\left(u_{j}\right) & \rightarrow \int \nabla a(\bar{\vartheta} x) \cdot(\bar{\vartheta} x) F(u) .
\end{aligned}
$$

Then, since $u_{j} \rightarrow u$ in $H^{s}\left(\mathbb{R}^{n}\right)$ as $j \rightarrow \infty$, we obtain

$$
\frac{n-2 s}{2} \int\left|(-\Delta)^{s / 2} u\right|^{2}=n \bar{\vartheta}^{2 s} \int\left(\left(a(\bar{\vartheta} x)+\frac{\nabla a(\bar{\vartheta} x) \cdot(\bar{\vartheta} x)}{n}\right) F(u)-\frac{\lambda u^{2}}{2}\right) .
$$

Hence $u(\cdot / \bar{\vartheta}) \in \mathcal{P}$ and, by uniqueness of the projection in $\mathcal{P}, \bar{\vartheta}=\vartheta_{2}(u)$.

Lemma 4.3. If $u \in \mathcal{P}_{\infty}$, then $\int G_{\infty}(u)>0$.

Proof. Let $u \in \mathcal{P}_{\infty}$. Of course $\int G_{\infty}(u) \geq 0$. Assume by contradiction that $\int G_{\infty}(u)=0$. Then

$$
0=\left\|(-\Delta)^{s / 2} u\right\|_{2}^{2}=\frac{C(n, s)}{2} \iint \frac{(u(x)-u(y))^{2}}{|x-y|^{n+2 s}} d x d y,
$$

so that $u$ is constant and hence, as $u \in L^{2}\left(\mathbb{R}^{n}\right), u=0$, contradicting $u \in \mathcal{P}_{\infty}$.

Lemma 4.4. If $u \in \mathcal{P}_{\infty}$, then there exists a unique $\vartheta>0$ such that $u(\cdot / \vartheta) \in \mathcal{P}$ and $\vartheta>1$.

Proof. Let $u \in \mathcal{P}_{\infty}$. Then, by Lemma 4.3, $\int G_{\infty}(u)>0$. In turn, by Lemma 4.1 , there exists a unique $\vartheta>0$ such that $u(\cdot / \vartheta) \in \mathcal{P}$. Now, we are left with the proof that $\vartheta>1$. By the arguments in the previous lemmas, it follows that $\vartheta$ satisfies

$$
\frac{n-2 s}{2} \int\left|(-\Delta)^{s / 2} u\right|^{2}=n \vartheta^{2 s} \int\left(\left(a(\vartheta x)+\frac{\nabla a(\vartheta x) \cdot(\vartheta x)}{n}\right) F(u)-\lambda \frac{u^{2}}{2}\right) .
$$

By condition $(A 4)$, we get

$$
\frac{n-2 s}{2 n} \int\left|(-\Delta)^{s / 2} u\right|^{2}<\vartheta^{2 s} \int\left(a_{\infty} F(u)-\lambda \frac{u^{2}}{2}\right)=\vartheta^{2 s} \int G_{\infty}(u) .
$$

Since $u \in \mathcal{P}_{\infty}$, the inequality above yields $\theta>1$. 
Lemma 4.5. If $u \in \mathcal{P}$, then $\int G_{\infty}(u)>0$.

Proof. Let $u \in \mathcal{P}$. Then, by condition $(A 4), u$ satisfies

$$
\frac{n-2 s}{2} \int\left|(-\Delta)^{s / 2} u\right|^{2}=n \int\left(\left(a(x)+\frac{\nabla a(x) \cdot x}{n}\right) F(u)-\lambda \frac{u^{2}}{2}\right)<n \int G_{\infty}(u) .
$$

Since $\int\left|(-\Delta)^{s / 2} u\right|^{2}>0$ otherwise $u$ would be constant and hence the zero function as $u \in L^{2}\left(\mathbb{R}^{n}\right)$, the assertion follows.

Lemma 4.6. If $u \in \mathcal{P}$, then there exists a unique $\vartheta>0$ such that $u(\cdot / \vartheta) \in \mathcal{P}_{\infty}$ and $\vartheta<1$.

Proof. Let $u \in \mathcal{P}$, then $\int G_{\infty}(u)>0$ by Lemma 4.5. By Lemma 4.1, there exists a unique $\vartheta>0$ such that $u(\cdot / \vartheta) \in \mathcal{P}_{\infty}$. We are left with the proof that $\vartheta<1$. Notice that

$$
\frac{n-2 s}{2 n} \int\left|(-\Delta)^{s / 2} u\right|^{2}<\int G_{\infty}(u) .
$$

Since $u(\cdot / \vartheta) \in \mathcal{P}_{\infty}$, then the assertion follows since $\vartheta>0$ satisfies

$$
\vartheta^{2 s}=\frac{n-2 s}{2 n} \frac{\int\left|(-\Delta)^{s / 2} u\right|^{2}}{\int G_{\infty}(u)}<1 .
$$

This concludes the proof.

Notice that, as a consequence of the previous results, a given function $u \in H^{s}\left(\mathbb{R}^{n}\right) \backslash\{0\}$ can be projected onto the manifolds $\mathcal{P}$ and $\mathcal{P}_{\infty}$ if and only if $\int G_{\infty}(u)>0$. We will also need the following

Lemma 4.7. If $u \in \mathcal{P}_{\infty}$, then $u(\cdot-y) \in \mathcal{P}_{\infty}$, for all $y \in \mathbb{R}^{n}$. Moreover, there exists $\vartheta_{y}>1$ with

$$
u\left(\frac{\cdot-y}{\vartheta_{y}}\right) \in \mathcal{P}, \quad \lim _{|y| \rightarrow \infty} \vartheta_{y}=1 .
$$

Proof. If $u \in \mathcal{P}_{\infty}$, then from translation invariance, we have $u(\cdot-y) \in \mathcal{P}_{\infty}$, for all $y \in \mathbb{R}^{n}$. Furthermore, from Lemma 4.4, there exists $\vartheta_{y}>1$ such that $u\left((\cdot-y) / \vartheta_{y}\right) \in \mathcal{P}$. Suppose by contradiction that there exists a sequence $\left(y_{j}\right) \subset \mathbb{R}^{n}$ with $\left|y_{j}\right| \rightarrow+\infty$ and $\vartheta_{y_{j}}$ converges either to $A>1$ or $+\infty$. Let us define

$$
K\left(\vartheta_{y_{j}} x+y_{j}\right):=a\left(\vartheta_{y_{j}} x+y_{j}\right)+\frac{\nabla a\left(\vartheta_{y_{j}} x+y_{j}\right) \cdot\left(\vartheta_{y_{j}} x+y_{j}\right)}{n} .
$$

From conditions (f1)-(f2) we have $0 \leq K\left(\vartheta_{y_{j}} x+y_{j}\right) F(u(x))<a_{\infty} F(u(x)) \leq C u^{2}(x)$ for a.e. $x \in \mathbb{R}^{n}$ and for some positive constant $C$. Hence, by Lebesgue Dominated Convergence Theorem, we get

$$
\lim _{j \rightarrow \infty} \int\left(K\left(\vartheta_{y_{j}} x+y_{j}\right) F(u)-\lambda \frac{u^{2}}{2}\right)=\int G_{\infty}(u) .
$$

But for each $y_{j}$ it follows that $u\left(\frac{\cdot-y_{j}}{\vartheta_{y_{j}}}\right) \in \mathcal{P}$ with $\vartheta_{y_{j}}>1$, which means we have

$$
\frac{n-2 s}{2} \int\left|(-\Delta)^{s / 2} u\right|^{2}=n \vartheta_{y_{j}}^{2 s} \int\left(K\left(\vartheta_{y_{j}} x+y_{j}\right) F(u)-\lambda \frac{u^{2}}{2}\right) .
$$

The right-hand side of formula (4.5) goes to $+\infty$ or to $n A^{2 s} \int G_{\infty}(u)$, while the left-hand side is constant. In the first case we immediately get a contradiction. In the second case, as $u \in \mathcal{P}_{\infty}$ and $A>1$, we get a contradiction too.

Under the assumption of Lemma 4.7, we have the following

Lemma 4.8. $\sup _{y \in \mathbb{R}^{n}} \vartheta_{y}=\bar{\vartheta}<+\infty$ and $\bar{\vartheta}>1$. 
Proof. From Lemma 4.7 there is $R>0$ such that $\left|\vartheta_{y}\right| \leq 2$ if $|y|>R$. There exists $M>0$ such that $\sup \left\{\vartheta_{y}:|y| \leq R\right\} \leq M$. In fact, suppose that there exists a sequence $\left(y_{j}\right)$ with $\left|y_{j}\right| \leq R$ such that $\vartheta_{y_{j}} \rightarrow+\infty$ as $j \rightarrow \infty$. As in the previous lemma, (4.4) holds. Therefore, from (4.5), it follows

$$
\frac{n-2 s}{2} \int\left|(-\Delta)^{s / 2} u\right|^{2}=n \vartheta_{y_{j}}^{2 s}\left(\int G_{\infty}(u)+o_{y_{j}}(1)\right)
$$

Since $\vartheta_{y_{j}} \rightarrow+\infty$ and the left-hand side is constant we get a contradiction and the proof is complete.

Lemma 4.9. There exists a real number $\hat{\sigma}>0$ such that $\inf _{u \in \mathcal{P}} \int\left|(-\Delta)^{s / 2} u\right|^{2} \geq \hat{\sigma}$.

Proof. Let $u \in \mathcal{P}$, then $u$ satisfies (3.1) and by condition $(A 4)$, we have

$$
0<\frac{n-2 s}{2} \int\left|(-\Delta)^{s / 2} u\right|^{2}<n \int\left(a_{\infty} F(u)-\lambda \frac{u^{2}}{2}\right)
$$

On the other hand, from condition (1.8) with $p=2 n /(n-2 s)$, given $0<\varepsilon<\frac{\lambda}{a_{\infty}}$, we get

$$
0<\frac{n-2 s}{2 n} \int\left|(-\Delta)^{s / 2} u\right|^{2}<a_{\infty} C\|u\|_{2 n /(n-2 s)}^{2 n /(n-2 s)}
$$

for some $C>0$. Using the fractional Sobolev inequality (cf. [10, Theorem 6.5$]$ ), we find $\hat{C}>0$ with

$$
0<\frac{n-2 s}{2 n a_{\infty} C \hat{C}}<\left(\int\left|(-\Delta)^{s / 2} u\right|^{2}\right)^{2 s /(n-2 s)}
$$

which yields the assertion with $\hat{\sigma}:=\left((n-2 s) /\left(2 n a_{\infty} C \hat{C}\right)\right)^{(n-2 s) / 2 s}>0$.

Lemma 4.10. $p=: \inf _{u \in \mathcal{P}} I(u)>0$.

Proof. Let $u \in \mathcal{P}$, then $I(u)$ satisfies

$$
I(u)=\frac{s}{n} \int\left|(-\Delta)^{s / 2} u\right|^{2}+\int \frac{\nabla a(x) \cdot x}{n} F(u) \geq \frac{s}{n} \int\left|(-\Delta)^{s / 2} u\right|^{2} \geq \frac{s \hat{\sigma}}{n}>0,
$$

by Lemma 4.9 and condition $(A 3)$. This concludes the proof.

If $u \in H^{s}\left(\mathbb{R}^{n}\right)$ with $\int G_{\infty}(u)>0$ and $\vartheta>0$ is such that $u(\cdot / \vartheta) \in \mathcal{P}_{\infty}$, then

$$
I_{\infty}(u(x / \vartheta))=\frac{s}{n} \vartheta^{n-2 s} \int\left|(-\Delta)^{s / 2} u\right|^{2} .
$$

Let $c_{\infty}$ be defined as in (2.3). Then, we have the following

Lemma 4.11. $p=c_{\infty}$.

Proof. Let $w \in H^{s}\left(\mathbb{R}^{n}\right)$ be a ground state solution to (2.1). Then $w \in \mathcal{P}_{\infty}$ and $I_{\infty}(w)=c_{\infty}$, by virtue of Theorem 2.1. Set $w_{y}:=w(x-y)$, for any $y \in \mathbb{R}^{n}$. Of course $w_{y} \in \mathcal{P}_{\infty}$ and $I_{\infty}\left(w_{y}\right)=c_{\infty}$, by translation invariance. From Lemma 4.4 we find a unique $\vartheta_{y}>1$ with $\tilde{w}_{y}=w_{y}\left(\cdot / \vartheta_{y}\right) \in \mathcal{P}$. 
Therefore, we have

$$
\begin{aligned}
& \left|I\left(\tilde{w}_{y}\right)-c_{\infty}\right|=\left|I\left(\tilde{w}_{y}\right)-I_{\infty}\left(w_{y}\right)\right| \\
& =\left.\left|\frac{1}{2} \int\right|(-\Delta)^{s / 2} \tilde{w}_{y}\right|^{2}-\int G\left(x, \tilde{w}_{y}\right)-\frac{1}{2} \int\left|(-\Delta)^{s / 2} w_{y}\right|^{2}+\int G_{\infty}\left(w_{y}\right) \mid \\
& =\left.\left|\frac{1}{2}\left(\vartheta_{y}^{n-2 s}-1\right) \int\right|(-\Delta)^{s / 2} w_{y}\right|^{2}-\int\left(a(x) F\left(\tilde{w}_{y}\right)-\frac{\lambda \tilde{w}_{y}^{2}}{2}\right)+\int\left(a_{\infty} F\left(w_{y}\right)-\frac{\lambda w_{y}^{2}}{2}\right) \mid \\
& =\left.\left|\frac{1}{2}\left(\vartheta_{y}^{n-2 s}-1\right) \int\right|(-\Delta)^{s / 2} w\right|^{2}-\vartheta_{y}^{n} \int\left(a\left(x \vartheta_{y}+y\right) F(w)-\frac{\lambda w^{2}}{2}\right)+\int\left(a_{\infty} F(w)-\frac{\lambda w^{2}}{2}\right) \mid \\
& =\left.\left|\frac{1}{2}\left(\vartheta_{y}^{n-2 s}-1\right) \int\right|(-\Delta)^{s / 2} w\right|^{2}+\left(\vartheta_{y}^{n}-1\right) \int \frac{\lambda w^{2}}{2}-\vartheta_{y}^{n} \int a\left(x \vartheta_{y}+y\right) F(w)+\int a_{\infty} F(w) \mid \\
& \leq \frac{\left|\vartheta_{y}^{n-2 s}-1\right|}{2} \int\left|(-\Delta)^{s / 2} w\right|^{2}+\left|\vartheta_{y}^{n}-1\right| \int \frac{\lambda w^{2}}{2}+\int|F(w)|\left|a_{\infty}-\vartheta_{y}^{n} a\left(x \vartheta_{y}+y\right)\right| .
\end{aligned}
$$

Since $\vartheta_{y} \rightarrow 1$ if $|y| \rightarrow+\infty$, we obtain

$|F(w)|\left|a_{\infty}-\vartheta_{y}^{n} a\left(x \vartheta_{y}+y\right)\right| \rightarrow 0 \quad$ as $|y| \rightarrow \infty$, a.e. in $\mathbb{R}^{n}, \quad|F(w)|\left|a_{\infty}-\vartheta_{y}^{n} a\left(x \vartheta_{y}+y\right)\right| \leq C|w|^{2}$,

for some positive constant $C$ independent of $y$. By Lebesgue Dominated Convergence Theorem,

$$
\int|F(w)|\left|a_{\infty}-\vartheta_{y}^{n} a\left(x \vartheta_{y}+y\right)\right|=o_{y}(1), \quad \text { as }|y| \rightarrow \infty .
$$

In turn, we conclude that $\left|I\left(\tilde{w}_{y}\right)-c_{\infty}\right| \leq o_{y}(1)$, as $|y| \rightarrow \infty$. Then, $p=\inf _{u \in \mathcal{P}} I(u) \leq c_{\infty}$. On the other hand, consider $u \in \mathcal{P}$ and let $0<\vartheta<1$ by Lemma 4.6 be such that $u(\cdot / \vartheta) \in \mathcal{P}_{\infty}$. Since $u \in \mathcal{P}$, then

$$
\begin{aligned}
I(u) & =\frac{s}{n} \int\left|(-\Delta)^{s / 2} u\right|^{2}+\frac{1}{n} \int \nabla a(x) \cdot x F(u)>\frac{s}{n} \int\left|(-\Delta)^{s / 2} u\right|^{2} \\
& \geq \frac{s}{n} \vartheta^{n-2 s} \int\left|(-\Delta)^{s / 2} u\right|^{2}=I_{\infty}(u(x / \vartheta)) \geq \inf _{u \in \mathcal{P}_{\infty}} I_{\infty}(u)=m=c_{\infty},
\end{aligned}
$$

in light of (4.7), (A3) and Lemma 2.4. Hence $p=\inf _{u \in \mathcal{P}} I(u) \geq c_{\infty}$, which concludes the proof.

Lemma 4.12. $\mathcal{P}$ is a natural constraint for (1.2).

Proof. If $u \in \mathcal{P}$ is a critical point of $\left.I\right|_{\mathcal{P}}$, there exists $\mu \in \mathbb{R}$ with $I^{\prime}(u)+\mu J^{\prime}(u)=0$. The proof is complete as soon as we show that $\mu=0$. Computing $I^{\prime}(u)(\varphi)+\mu J^{\prime}(u)(\varphi)$ for any $\varphi \in H^{s}\left(\mathbb{R}^{n}\right)$ yields

$$
\begin{aligned}
0 & =\int(-\Delta)^{s / 2} u(-\Delta)^{s / 2} \varphi+\lambda u \varphi-\int a(x) f(u) \varphi \\
& +\mu\left[(n-2 s) \int(-\Delta)^{s / 2} u(-\Delta)^{s / 2} \varphi-n \int\left(\left(a(x)+\frac{\nabla a(x) \cdot x}{n}\right) f(u) \varphi-\lambda u \varphi\right)\right] .
\end{aligned}
$$

so that $u$ satisfies the equation

$$
(1+\mu(n-2 s))(-\Delta)^{s} u+\lambda(1+\mu n) u=[(1+\mu n) a(x)+\mu \nabla a(x) \cdot x] f(u) .
$$

The solutions of this equation satisfy a Pohožaev identity $Q(u)=0$, where

$$
Q(u)=\frac{(1+\mu(n-2 s))(n-2 s)}{2} \int\left|(-\Delta)^{s / 2} u\right|^{2}-n \int \widehat{G}(x, u)-\int x \cdot \widehat{G}_{x}(x, u),
$$


where we have

$$
\begin{aligned}
\widehat{G}(x, u) & =((1+\mu n) a(x)+\mu \nabla a(x) \cdot x) F(u)-\lambda \frac{(1+\mu n)}{2} u^{2}, \\
x \cdot \widehat{G}_{x}(x, u) & =\left((1+\mu+\mu n) \nabla a(x) \cdot x+\mu x \cdot \mathcal{H}_{a}(x) \cdot x\right) F(u) .
\end{aligned}
$$

Therefore, $Q$ rewrites as follows

$$
\begin{aligned}
Q(u) & \left.=\frac{(1+\mu(n-2 s))(n-2 s)}{2} \int \mid(-\Delta)^{s / 2} u\right)\left.\right|^{2} \\
& -n \int((1+\mu n) a(x)+\mu \nabla a(x) \cdot x) F(u)-\lambda \frac{(1+\mu n)}{2} u^{2} \\
& -\int\left((1+\mu+\mu n) \nabla a(x) \cdot x+\mu x \cdot \mathcal{H}_{a}(x) \cdot x\right) F(u) \\
& =\frac{(1+\mu(n-2 s))(n-2 s)}{2} \int\left|(-\Delta)^{s / 2} u\right|^{2} \\
& -n(1+\mu n) \int\left(a(x)+\frac{\nabla a(x) \cdot x}{n}\right) F(u)-\lambda \frac{u^{2}}{2} \\
& -(n+1) \mu \int\left(\nabla a(x) \cdot x+\frac{x \cdot \mathcal{H}_{a}(x) \cdot x}{n+1}\right) F(u) .
\end{aligned}
$$

Recalling that $u \in \mathcal{P}$ and substituting (3.1) in the equation above, it follows that

$$
\begin{aligned}
Q(u) & =\frac{(1+\mu(n-2 s))(n-2 s)}{2} \int\left|(-\Delta)^{s / 2} u\right|^{2}-(1+\mu n) \frac{(n-2 s)}{2} \int\left|(-\Delta)^{s / 2} u\right|^{2} \\
& -(n+1) \mu \int\left(\nabla a(x) \cdot x+\frac{x \cdot \mathcal{H}_{a}(x) \cdot x}{n+1}\right) F(u) \\
& =-\mu s(n-2 s) \int\left|(-\Delta)^{s / 2} u\right|^{2}-(n+1) \mu \int\left(\nabla a(x) \cdot x+\frac{x \cdot \mathcal{H}_{a}(x) \cdot x}{n+1}\right) F(u) .
\end{aligned}
$$

On the other hand, since $u$ satisfies $Q(u)=0$, we end up with

$$
-\mu s(n-2 s) \int\left|(-\Delta)^{s / 2} u\right|^{2}=(n+1) \mu \int\left(\nabla a(x) \cdot x+\frac{x \cdot \mathcal{H}_{a}(x) \cdot x}{n+1}\right) F(u) .
$$

From $(A 5)$ we have that, if $\mu>0$, the right-hand side of the equation is nonnegative as

$$
\nabla a(x) \cdot x+\frac{x \cdot \mathcal{H}_{a}(x) \cdot x}{n+1} \geq \frac{n}{n+1}\left(\nabla a(x) \cdot x+\frac{x \cdot \mathcal{H}_{a}(x) \cdot x}{n}\right) \geq 0,
$$

while the left-hand side is negative. If $\mu<0$ one gets the same contradiction. Whence $\mu=0$.

Proof of Theorem 1.1 concluded. Assume by contradiction that there exists a critical point $z \in$ $H^{s}\left(\mathbb{R}^{n}\right)$ of $I$ at level $p$. In particular, $z \in \mathcal{P}$ and $I(z)=p$. Let $\vartheta \in(0,1)$ be such that $z(\cdot / \vartheta) \in \mathcal{P}_{\infty}$. Then

$$
\begin{aligned}
p=I(z) & =\frac{s}{n} \int\left|(-\Delta)^{s / 2} z\right|^{2}+\frac{1}{n} \int \nabla a(x) \cdot x F(z) \\
& >\frac{s}{n} \int\left|(-\Delta)^{s / 2} z\right|^{2}>\frac{s}{n} \vartheta^{n-2 s} \int\left|(-\Delta)^{s / 2} z\right|^{2} \\
& =I_{\infty}(z(\cdot / \vartheta)) \geq \inf _{u \in \mathcal{P}_{\infty}} I_{\infty}(u)=m=c_{\infty},
\end{aligned}
$$

using (A3) and (4.7), Lemma 2.4 and Theorem 2.1. Then $p>c_{\infty}$, contradicting Lemma 4.11. In particular the infimum $p$ is not achieved, otherwise, if $I(v)=p$ and $\left.I^{\prime}\right|_{\mathcal{P}}(v)=0$ for some $v \in H^{s}\left(\mathbb{R}^{n}\right)$, in light of Lemma 4.12, we would have $I^{\prime}(v)=0$, contradicting the first part of Theorem 1.1. 


\section{EXISTENCE RESULTS}

In this section we show the existence of a solution of problem (1.2). To this aim, we shall assume that the hypotheses of Theorem 1.2 are satisfied. As we have seen in the previous sections, we should look for solutions which have energy levels above $c_{\infty}$. In order to find such a solution we follow some ideas of [1] based upon linking and the barycenter function on the Nehari manifold. In our case, since the nonlinear terms of the equation are not homogeneous, we are led to the Pohožaev manifold $\mathcal{P}$ and obtain the desired solution by a linking argument. We also make use of a barycenter function, similar to that of [1] and used by G.S. Spradlin [25, 26] as well.

Lemma 5.1. I satisfies the geometrical properties of the Mountain Pass theorem.

Proof. On one hand, for the local minimum condition at the origin, by (1.8) one can argue exactly as in the proof of Lemma 2.3. On the other hand, if $w \in H^{s}\left(\mathbb{R}^{n}\right)$ is a least energy solution to (2.1), by Lemma 2.2 there exists $\gamma \in \Gamma_{\infty}$ such that $\gamma(t)=w(x / t L)$ for $t>0$ and $L>0$ large enough. In turn, if $\gamma_{y}(t):=w((\cdot-y) / t L)$, by $(A 2)$ and Lebesgue Dominated Convergence Theorem,

$$
I\left(\gamma_{y}(1)\right)=I_{\infty}\left(\gamma_{y}(1)\right)+\int\left(a_{\infty}-a(x+y)\right) F(\gamma(1))=I_{\infty}(\gamma(1))+o_{y}(1)<0, \quad \text { for }|y| \text { large },
$$

since $I_{\infty}(\gamma(1))<0$, concluding the proof.

Let $c$ be the min-max mountain pass level for $I$

$$
c=\min _{\gamma \in \Gamma} \max _{t \in[0,1]} I(\gamma(t)), \quad \Gamma:=\left\{\gamma \in C\left([0,1], H^{s}\left(\mathbb{R}^{n}\right)\right): \gamma(0)=0, I(\gamma(1))<0\right\} .
$$

We start by proving that the min-max levels of the Mountain Pass Theorem for $I$ and $I_{\infty}$ agree.

Lemma 5.2. $c_{\infty}=c$.

Proof. If $\gamma \in \Gamma$, then $I(\gamma(1))<0$ and since $I_{\infty} \leq I$, we have $I_{\infty}(\gamma(1))<0$. Then, $\Gamma \subset \Gamma_{\infty}$ yielding

$$
c_{\infty}=\inf _{\gamma \in \Gamma_{\infty}} \max _{t \in[0,1]} I_{\infty}(\gamma(t)) \leq \inf _{\gamma \in \Gamma} \max _{t \in[0,1]} I_{\infty}(\gamma(t)) \leq \inf _{\gamma \in \Gamma} \max _{t \in[0,1]} I(\gamma(t))=c .
$$

Let now $\varepsilon>0$ be arbitrary and let $\gamma \in \Gamma_{\infty}$ such that $I_{\infty}(\gamma(t)) \leq c_{\infty}+\varepsilon$, for all $t \in[0,1]$. Choose $y \in \mathbb{R}^{n}$ and translating $\tau_{y}(\gamma(t))(x):=\gamma(t)(x-y)$ with $|y|$ large enough, we get $\tau_{y} \circ \gamma \in \Gamma$ (see Lemma 5.1). If $t_{y} \in[0,1]$ is such that $I\left(\tau_{y}\left(\gamma\left(t_{y}\right)\right)\right)$ is the maximum value on $[0,1]$ of $t \mapsto I\left(\tau_{y} \circ \gamma(t)\right)$, then

$$
c_{\infty}+\varepsilon \geq I_{\infty}\left(\gamma\left(t_{y}\right)\right)=I_{\infty}\left(\tau_{y} \circ \gamma\left(t_{y}\right)\right)=\max _{[0,1]} I\left(\tau_{y} \circ \gamma\right) \geq \inf _{\gamma \in \Gamma} \max _{t \in[0,1]} I(\gamma(t))=c .
$$

This gives $c_{\infty} \geq c$ by the arbitrariness of $\varepsilon$ and the assertion follows.

Lemma 5.3. $p=c$.

Proof. The assertion follows by combining Lemma 4.11 and Lemma 5.2.

Now we observe the following property of $\mathcal{P}$ with respect to the paths in the Mountain Pass Theorem.

Lemma 5.4. For every $\gamma \in \Gamma$ there exists $s \in(0,1)$ such that $\gamma(s) \in \mathcal{P}$.

Proof. By the proof of Lemma $3.1(a)$, we learn that there exists $\rho>0$ such that $J(u)>0$ if $0<\|u\|_{H^{s}}<\rho$. Furthermore, we have

$$
\begin{aligned}
J(u) & =\frac{n-2 s}{2} \int\left|(-\Delta)^{s / 2} u\right|^{2}-n \int G(x, u)-\int \nabla a(x) \cdot x F(u) \\
& =n I(u)-s \int\left|(-\Delta)^{s / 2} u\right|^{2}-\int \nabla a(x) \cdot x F(u) .
\end{aligned}
$$

From $(A 3)$ it follows that $J(u)<n I(u)$, for every $u \in H^{s}\left(\mathbb{R}^{n}\right) \backslash\{0\}$. If $\gamma \in \Gamma$, we have $J(\gamma(0))=0$ and $J(\gamma(1))<n I(\gamma(1))<0$. Then there exists $t \in(0,1)$ with $\|\gamma(t)\|_{H^{s}}>\rho$ and $J(\gamma(t))=0$. 
We recall that a sequence $\left(u_{j}\right)$ is said to be a Cerami sequence for $I$ at level $d$ in $\mathbb{R}$, denoted by $(C e)_{d}$, if $I\left(u_{n}\right) \rightarrow d$ and $\left\|I^{\prime}\left(u_{j}\right)\right\|_{H^{-s}}\left(1+\left\|u_{j}\right\|_{H^{s}}\right) \rightarrow 0$. We have the following

Lemma 5.5. If $\left(u_{j}\right)$ is a $(C e)_{d}$ sequence with $d>0$, then it has a bounded subsequence.

Proof. By contradiction, let $\left\|u_{j}\right\|_{H^{s}} \rightarrow+\infty$. If $\hat{u}_{j}:=u_{j}\left\|u_{j}\right\|_{H^{s}}^{-1}$, then $\left\|\hat{u}_{j}\right\|_{H^{s}}=1$ and $\hat{u}_{j} \rightarrow \hat{u}$, up to a subsequence. Therefore, one of the two cases occur:

$$
\begin{aligned}
& \text { Case 1: } \quad \limsup _{j \rightarrow \infty} \sup _{y \in \mathbb{R}^{n}} \int_{B_{1}(y)}\left|\hat{u}_{j}\right|^{2}=\delta>0, \\
& \text { Case 2: } \quad \limsup _{j \rightarrow \infty} \sup _{y \in \mathbb{R}^{n}} \int_{B_{1}(y)}\left|\hat{u}_{j}\right|^{2}=0 .
\end{aligned}
$$

Suppose Case 2 hold. Fixing $L>2 \sqrt{d D}$, with $D$ as in assumption (f3), gives

$$
I\left(L u_{j}\left\|u_{j}\right\|_{H^{s}}^{-1}\right)=\frac{L^{2}}{2}-\int a(x) F\left(L u_{j}\left\|u_{j}\right\|_{H^{s}}^{-1}\right) .
$$

Given $\varepsilon>0$, by inequality (1.8) there exists $C_{\varepsilon}>0$ (here $2<p<2 n /(n-2 s)$ ) with

$$
\int a(x) F\left(L u_{j}\left\|u_{j}\right\|_{H^{s}}^{-1}\right)<\frac{a_{\infty} \varepsilon L^{2}}{2} \frac{\left\|u_{j}\right\|_{2}^{2}}{\lambda\left\|u_{j}\right\|_{2}^{2}+\left\|(-\Delta)^{s / 2} u_{j}\right\|_{2}^{2}}+C_{\varepsilon} L^{p}\left\|\hat{u}_{j}\right\|_{p}^{p} \leq \frac{a_{\infty} \varepsilon L^{2}}{2 \lambda}+o_{j}(1),
$$

where $\left\|\hat{u}_{j}\right\|_{p} \rightarrow 0$ by a variant of Lions' Lemma [19, Lemma I.1]. For $\varepsilon=\lambda /\left(2 a_{\infty}\right)$, we have

$$
I\left(L u_{j}\left\|u_{j}\right\|_{H^{s}}^{-1}\right) \geq \frac{L^{2}}{4}-o_{j}(1) .
$$

We have $L\left\|u_{j}\right\|_{H^{s}}^{-1} \in(0,1)$ for $j$ large and if we consider $t_{j} \in(0,1)$ with $I\left(t_{j} u_{j}\right)=\max _{t \in[0,1]} I\left(t u_{j}\right)$,

$$
I\left(t_{j} u_{j}\right)=\max _{t \in[0,1]} I\left(t u_{j}\right) \geq I\left(L u_{j}\left\|u_{j}\right\|_{H^{s}}^{-1}\right) \geq \frac{L^{2}}{4}-o_{j}(1) .
$$

On the other hand, using ( $f 3)$ we obtain

$$
\begin{aligned}
I\left(t_{j} u_{j}\right) & =I\left(t_{j} u_{j}\right)-\frac{1}{2} I^{\prime}\left(t_{j} u_{j}\right)\left(t_{j} u_{j}\right)=\int a(x)\left(\frac{1}{2} f\left(t_{j} u_{j}\right)\left(t_{j} u_{j}\right)-F\left(t_{j} u_{j}\right)\right) \\
& \left.\leq D \int a(x)\left(\frac{1}{2} f\left(u_{j}\right) u_{j}-F\left(u_{j}\right)\right)=D\left(I\left(u_{j}\right)-\frac{1}{2} I^{\prime}\left(u_{j}\right) u_{j}\right)\right)=D d+o_{j}(1) .
\end{aligned}
$$

Then, on account of the choice of $L$, combining (5.2) and (5.3), we get a contradiction. In Case 1, let $\left(y_{j}\right)$ be a sequence such that $\left|y_{j}\right| \rightarrow+\infty$ and

$$
\int_{B_{1}\left(y_{j}\right)}\left|\hat{u}_{j}\right|^{2}>\delta / 2
$$

Recalling that $\hat{u}_{j}\left(\cdot+y_{j}\right) \rightarrow \bar{u}$ in $H^{s}\left(\mathbb{R}^{n}\right)$ as $j \rightarrow \infty$, we obtain $\int_{B_{1}(0)}|\bar{u}(x)|^{2}>\delta / 2$, namely $\bar{u} \neq 0$. Thus, there exists $\Omega \subset B_{1}(0)$, with $|\Omega|>0$ such that

$$
0 \neq \bar{u}(x)=\lim _{j \rightarrow \infty} \hat{u}_{j}\left(x+y_{j}\right)=\lim _{j \rightarrow \infty} \frac{u_{j}\left(x+y_{j}\right)}{\left\|u_{j}\right\|_{H^{s}}}, \quad \text { a.e. } x \in \Omega,
$$

yielding $u_{j}\left(x+y_{j}\right) \rightarrow \infty$ for a.e. $x \in \Omega$. We claim that, actually $u_{j}\left(x+y_{j}\right) \rightarrow+\infty$ for $x \in \Omega$. Setting $\zeta_{j}(x):=\hat{u}_{j}\left(x+y_{j}\right)$, for a $\mu_{j} \rightarrow 0$ in $H^{-s}\left(\mathbb{R}^{n}\right)$ as $j \rightarrow \infty$, we have

$$
(-\Delta)^{s / 2} \zeta_{j}+\lambda \zeta_{j}=\frac{a\left(x+y_{j}\right)}{\left\|u_{j}\right\|_{H^{s}}} f\left(\left\|u_{j}\right\|_{H^{s}} \zeta_{j}\right)+\frac{\mu_{j}}{\left\|u_{j}\right\|_{H^{s}}} .
$$


Testing this equation by $\zeta_{j}^{-}$and taking unto account that

$$
\int \frac{a\left(x+y_{j}\right)}{\left\|u_{j}\right\|_{H^{s}}} f\left(\left\|u_{j}\right\|_{H^{s}} \zeta_{j}\right) \zeta_{j}^{-}=0, \quad \frac{\left\langle\mu_{j}, \zeta_{j}^{-}\right\rangle}{\left\|u_{j}\right\|_{H^{s}}}=\frac{\left\langle\mu_{j}, u_{j}^{-}\left(\cdot+y_{j}\right)\right\rangle}{\left\|u_{j}\right\|_{H^{s}}^{2}}=o_{j}(1),
$$

by arguing as around formula (1.10), we conclude that $\left\|\zeta_{j}^{-}\right\|_{H^{s}}=o_{j}(1)$ as $j \rightarrow \infty$, hence in particular by the fractional Sobolev embedding $\left\|\zeta_{j}^{-}\right\|_{L^{p}}=o_{j}(1)$ as $j \rightarrow \infty$ for any $2 \leq p \leq 2 n /(n-2 s)$. Since $\zeta_{j}=\hat{u}_{j}\left(\cdot+y_{j}\right) \rightarrow \bar{u}$ in $L^{p}(\Omega)$, we also have $\zeta_{j}^{-}=\hat{u}_{j}^{-}\left(\cdot+y_{j}\right) \rightarrow \bar{u}^{-}$in $L^{p}(\Omega)$. But then $\bar{u}^{-}=0$ on $\Omega$ which means $\bar{u}>0$ on $\Omega$. In turn, from 5.5, we have the claim. Thus, by (f3), Fatou Lemma and $(A 1)$, with $\sigma:=\inf _{\mathbb{R}^{n}} a$,

$$
\begin{aligned}
& \liminf _{j \rightarrow \infty} \int a(x)\left(\frac{1}{2} f\left(u_{j}\right) u_{j}-F\left(u_{j}\right)\right) \\
& =\liminf _{j \rightarrow \infty} \int a\left(x+y_{j}\right)\left(\frac{1}{2} f\left(u_{j}\left(x+y_{j}\right)\right) u_{j}\left(x+y_{j}\right)-F\left(u_{j}\left(x+y_{j}\right)\right)\right. \\
& \geq \liminf _{j \rightarrow \infty} \int_{\Omega} \sigma\left(\frac{1}{2} f\left(u_{j}\left(x+y_{j}\right)\right) u_{j}\left(x+y_{j}\right)-F\left(u_{j}\left(x+y_{j}\right)\right)\right. \\
& \geq \int_{\Omega} \liminf _{j \rightarrow \infty} \sigma\left(\frac{1}{2} f\left(u_{j}\left(x+y_{j}\right)\right) u_{j}\left(x+y_{j}\right)-F\left(u_{j}\left(x+y_{j}\right)\right)=+\infty .\right.
\end{aligned}
$$

On the other hand, $\left|I^{\prime}\left(u_{j}\right) u_{j}\right| \leq\left\|I^{\prime}\left(u_{j}\right)\right\|_{H^{-s}}\left\|u_{j}\right\|_{H^{s}} \rightarrow 0$, as $j \rightarrow \infty$. Then,

$$
\int a(x)\left(\frac{1}{2} f\left(u_{j}\right) u_{j}-F\left(u_{j}\right)\right)=I\left(u_{j}\right)-\frac{1}{2} I^{\prime}\left(u_{j}\right) u_{j}=d+o_{j}(1),
$$

which gives a contradiction. If, instead, $\left(y_{j}\right)$ in (5.4) is bounded, say $\left|y_{j}\right| \leq R$ for some $R$, we obtain

$$
\frac{\delta}{2} \leq \int_{B_{1}\left(y_{j}\right)}\left|\hat{u}_{j}\right|^{2} \leq \int_{B_{2 R}(0)}\left|\hat{u}_{j}\right|^{2},
$$

and since $\hat{u}_{j} \rightarrow \hat{u}$ in $L^{2}\left(B_{2 R}(0)\right)$, it follows that

$$
\delta / 2 \leq \int_{B_{2 R}(0)}|\hat{u}|^{2} .
$$

Similarly to the previous case, there exists $\Omega \subset B_{2 R}(0)$ of positive measure such that (5.5) holds. The argument follows as above for the case where $\left(y_{j}\right)$ is unbounded and we get a contradiction.

The next step is to show the existence of a Cerami sequence for the functional $I$ at level $c$.

Lemma 5.6. Let $c$ be as in (5.1), then there exists a $(C e)_{c}$ sequence $\left(u_{n}\right) \subset H^{s}\left(\mathbb{R}^{n}\right)$.

Proof. We apply the Ghoussoub-Preiss theorem [11, Theorem 6] with $X=H^{s}\left(\mathbb{R}^{n}\right)$, see also [15]. Consider $z_{0}=0$ and $z_{1}$ in $H^{s}\left(\mathbb{R}^{n}\right)$ with $I\left(z_{1}\right)<0$ (cf. Lemma 5.1). Then the Pohožaev manifold $\mathcal{P}$ separates $z_{0}$ and $z_{1}$. Indeed, observe that $z_{0}=0 \notin \mathcal{P}$ and $z_{1} \notin \mathcal{P}$, since $J\left(z_{1}\right)<n I\left(z_{1}\right)<0$ (cf. proof of (a) of Lemma 5.4). Moreover, there exists $\rho>0$ such that, if $0<\|u\|_{H^{s}}<\rho$, then $J(u)>0$ (cf. proof of Lemma 3.1). We have $H^{s}\left(\mathbb{R}^{n}\right) \backslash \mathcal{P}=\{0\} \cup\{J>0\} \cup\{J<0\}$. The ball $B_{\rho}\left(z_{0}\right)$ is in a connected component $C_{1}$ of $\{0\} \cup\{J>0\}$. On the other hand, $z_{1}$ is in a connected component of $\{J<0\}$. In this setting, we get a sequence $\left(u_{j}\right) \subset H^{s}\left(\mathbb{R}^{n}\right)$ such that

$$
\delta\left(u_{j}, \mathcal{P}\right) \rightarrow 0, \quad I\left(u_{j}\right) \rightarrow c, \quad\left\|I^{\prime}\left(u_{j}\right)\right\|\left(1+\left\|u_{j}\right\|_{H^{s}}\right) \rightarrow 0,
$$

where $\delta$ denotes the geodesic metric on $H^{s}\left(\mathbb{R}^{n}\right)$, defined by

$$
\delta(u, v):=\inf \left\{\int_{0}^{1} \frac{\left\|\gamma^{\prime}(\sigma)\right\|_{H^{s}}}{1+\|\gamma(\sigma)\|_{H^{s}}} d \sigma: \gamma \in C^{1}\left([0,1], H^{s}\left(\mathbb{R}^{n}\right)\right), \gamma(0)=u, \gamma(1)=v\right\} .
$$

This complets the proof. 
For the following type of properties, we refer the reader to the book [29].

Lemma 5.7. Let $\left(u_{j}\right) \in H^{s}\left(\mathbb{R}^{n}\right)$ be a bounded sequence such that

$$
I\left(u_{j}\right) \rightarrow d>0 \text { and }\left\|I^{\prime}\left(u_{j}\right)\right\|_{H^{-s}}\left(1+\left\|u_{j}\right\|_{H^{s}}\right) \rightarrow 0 .
$$

Replacing $\left(u_{j}\right)$ by a subsequence, if necessary, there exists a solution $\bar{u}$ of (1.2), a number $k \in$ $\mathbb{N} \cup\{0\}, k$ functions $u^{1}, u^{2}, \ldots, u^{k}$ and $k$ sequences of points $y_{j}^{1}, y_{j}^{2}, \ldots, y_{j}^{k} \in \mathbb{R}^{n}$, satisfying:

a) $u_{j} \rightarrow \bar{u}$ in $H^{s}\left(\mathbb{R}^{n}\right)$ or

b) $u^{i} \in H^{s}\left(\mathbb{R}^{n}\right)$ are positive solutions to (2.1) radially symmetric about some point;

c) $\left|y_{n}^{i}\right| \rightarrow+\infty$ and $\left|y_{n}^{i}-y_{n}^{m}\right| \rightarrow+\infty, i \neq m$;

d) $u_{j}-\sum_{i=1}^{k} u^{i}\left(x-y_{j}^{i}\right) \rightarrow \bar{u}$;

e) $I\left(u_{j}\right) \rightarrow I(\bar{u})+\sum_{i=1}^{k} I_{\infty}\left(u^{i}\right)$

That the solutions $u^{i} \in H^{s}\left(\mathbb{R}^{n}\right)$ to $(2.1)$ are positive and radially symmetric about some point follows from [12, Theorem 1.3], namely a Gidas-Ni-Niremberg type result in the fractional case $\left(u^{i} \neq 0, u^{i} \geq 0\right.$ and hence $u^{i}>0$, see [12]).

Corollary 5.8. If $I\left(u_{j}\right) \rightarrow c_{\infty}$ and $\left\|I^{\prime}\left(u_{j}\right)\right\|_{H^{-s}}\left(1+\left\|u_{j}\right\|_{H^{s}}\right) \rightarrow 0$, then either $\left(u_{j}\right)$ is relatively compact in $H^{s}\left(\mathbb{R}^{n}\right)$ or Lemma 5.7 holds with $k=1$ and $\bar{u}=0$.

Let us set

$$
c_{\sharp}:=\inf \left\{c>c_{\infty}: c \text { is a radial critical value of } I_{\infty}\right\} .
$$

Then we have the following

Lemma 5.9. Assume that

$$
c_{\infty} \text { is an isolated radial critical level for } I_{\infty},
$$

Then $c_{\sharp}>c_{\infty}$ and I satisfies condition $(C e)$ at level $d \in\left(c_{\infty}, \min \left\{c_{\sharp}, 2 c_{\infty}\right\}\right)$. Assume now that

$$
\text { the limiting problem (2.1) admits a unique positive radial solution. }
$$

Then I satisfies condition $(C e)$ at level $d \in\left(c_{\infty}, 2 c_{\infty}\right)$.

Proof. Take a sequence $\left(u_{j}\right) \in H^{s}\left(\mathbb{R}^{n}\right)$ such that $I\left(u_{j}\right) \rightarrow d$ and $\left\|I^{\prime}\left(u_{j}\right)\right\|_{H^{-s}}\left(1+\left\|u_{j}\right\|_{H^{s}}\right) \rightarrow 0$ as $j \rightarrow \infty$. By Lemma 5.5, $\left(u_{j}\right)$ has a bounded subsequence. Applying Lemma 5.7, up to subsequences, we have

$$
u_{j}-\sum_{i=1}^{k} u^{i}\left(x-y_{j}^{i}\right) \rightarrow \bar{u} \quad \text { in } H^{s}\left(\mathbb{R}^{n}\right), \quad I\left(u_{j}\right) \rightarrow I(\bar{u})+\sum_{i=1}^{k} I_{\infty}\left(u^{i}\right),
$$

where $u^{i}$ is a solution to (2.1), $\left|y_{j}^{i}\right| \rightarrow+\infty$ and $\bar{u}$ is a (possibly zero) solution of (1.2). Since $d<2 c_{\infty}$, then $k<2$. If $k=1$, we have two cases to distinguish.

Let us first assume that (5.6) holds. Then $c_{\sharp}>c_{\infty}$, otherwise there exists a sequence $c_{j}$ of radially symmetric (about some point) critical values of $I_{\infty}$ such that $c_{j}>c_{\infty}$ and $c_{j} \rightarrow c_{\infty}$ as $j \rightarrow \infty$.

- $\bar{u} \neq 0$, which implies $I(\bar{u}) \geq p=c_{\infty}$ and hence $I\left(u_{j}\right) \geq 2 c_{\infty}$.

- $\bar{u}=0$, which yields $I\left(u_{j}\right) \rightarrow I_{\infty}\left(u_{1}\right)$. If $I_{\infty}\left(u_{1}\right)=c_{\infty}$, we have a contradiction. If $I_{\infty}\left(u_{1}\right)=\tilde{c}>$ $c_{\infty}$, then $I_{\infty}\left(u_{1}\right) \geq c_{\sharp} \geq \min \left\{c_{\sharp}, 2 c_{\infty}\right\}$, against $d<\min \left\{c_{\sharp}, 2 c_{\infty}\right\}$. Then $k=0$ and $u_{j} \rightarrow \bar{u}$.

Let us now assume that (5.7) holds.

- $\bar{u} \neq 0$, which implies $I(\bar{u}) \geq p=c_{\infty}$ and hence $I\left(u_{j}\right) \geq 2 c_{\infty}$.

- $\bar{u}=0$, which yields $I\left(u_{j}\right) \rightarrow I_{\infty}\left(u_{1}\right)=c_{\infty}$. The fact that $I_{\infty}\left(u_{1}\right)=c_{\infty}$ follows by using uniqueness assumption (5.7). These conclusions go against the assumption $c_{\infty}<d<2 c_{\infty}$. 
Lemma 5.10. Let $I\left(u_{j}\right) \rightarrow d>0$ and $\left\{u_{j}\right\} \subset \mathcal{P}$. Then $\left\{u_{j}\right\}$ is bounded in $H^{s}\left(\mathbb{R}^{n}\right)$.

Proof. If $u_{j} \in \mathcal{P}$, then using $(A 3)$ and the first equality of (4.6), we get

$$
d+1 \geq I\left(u_{j}\right) \geq \frac{s}{n} \int\left|(-\Delta)^{s / 2} u_{j}\right|^{2} .
$$

In turn, by the fractional Sobolev inequality, the sequence $\left\|u_{j}\right\|_{2 n /(n-2 s)}$ is also bounded. By (1.8) with $\varepsilon<\lambda /\|a\|_{\infty}$, we have

$$
\int a(x) F\left(u_{j}\right) \leq \frac{1}{2} \varepsilon\|a\|_{\infty}\left\|u_{j}\right\|_{2}^{2}+C_{\varepsilon}\left\|u_{j}\right\|_{2 n /(n-2 s)}^{2 n /(n-2 s)} .
$$

Replacing this in the expression of $I$

$$
d+1 \geq I\left(u_{j}\right) \geq \frac{1}{2} \int\left|(-\Delta)^{s / 2} u_{j}\right|^{2}+\frac{1}{2}\left(\lambda-\varepsilon\|a\|_{\infty}\right)\left\|u_{j}\right\|_{2}^{2}-C_{\varepsilon}\left\|u_{j}\right\|_{2 n /(n-2 s)}^{2 n /(n-2 s)},
$$

so $\left\|u_{j}\right\|_{2}$ is bounded as well, and the assertion follows.

Next, we introduce the barycenter function.

Definition 5.11. Define the barycenter function of a $u \in H^{s}\left(\mathbb{R}^{n}\right) \backslash\{0\}$ by setting

$$
\mu(u)(x):=\frac{1}{\left|B_{1}\right|} \int_{B_{1}(x)}|u(y)| d y .
$$

It follows that $\mu(u) \in L^{\infty}\left(\mathbb{R}^{n}\right) \cap C\left(\mathbb{R}^{n}\right)$. Subsequently, take

$$
\hat{u}(x):=\left[\mu(u)(x)-\frac{1}{2} \max \mu(u)\right]^{+} .
$$

It follows that $\hat{u} \in C_{0}\left(\mathbb{R}^{n}\right)$. Now define the barycenter of $u$ by

$$
\beta(u)=\frac{1}{\|\hat{u}\|_{L^{1}}} \int x \hat{u}(x) d x \in \mathbb{R}^{n} .
$$

Since $\hat{u}$ has compact support, by definition, $\beta(u)$ is well defined. $\beta$ satisfies the following properties:

(a) $\beta$ is a continuous function in $H^{s}\left(\mathbb{R}^{n}\right) \backslash\{0\}$.

(b) If $u$ is radially symmetric, then $\beta(u)=0$.

(c) Given $y \in \mathbb{R}^{n}$ and setting $u_{y}(x):=u(x-y)$, then $\beta\left(u_{y}\right)=\beta(u)+y$.

We shall also need the following

Lemma 5.12. Assume that $u_{j}, v_{j} \subset H^{s}\left(\mathbb{R}^{n}\right)$ are such that $\left\|u_{j}-v_{j}\right\|_{H^{s}} \rightarrow 0$ and $I^{\prime}\left(v_{j}\right) \rightarrow 0$ as $j \rightarrow \infty$. Then, $I^{\prime}\left(u_{j}\right) \rightarrow 0$ as $j \rightarrow \infty$

Proof. By assumption (1) of Theorem 1.2, we have $f \in \operatorname{Lip}\left(\mathbb{R}, \mathbb{R}^{+}\right)$. Observe first that, for every $w, \varphi, \psi \in H^{s}\left(\mathbb{R}^{n}\right)$, we have

$$
I^{\prime \prime}(w)(\varphi, \psi)=\int(-\Delta)^{s / 2} \varphi(-\Delta)^{s / 2} \psi+\lambda \int \varphi \psi-\int a(x) f^{\prime}(w) \varphi \psi .
$$

Also, by the Mean Value Theorem, for any $u, v \in H^{s}\left(\mathbb{R}^{n}\right)$ and $\varphi \in H^{s}\left(\mathbb{R}^{n}\right)$, there exists $\xi \in(0,1)$ with

$$
I^{\prime}(v)(\varphi)-I^{\prime}(u)(\varphi)=I^{\prime \prime}(u+\xi(v-u))(\varphi, v-u)
$$


Therefore, by taking into account that $\left|f^{\prime}\left(u_{j}+\xi_{j}\left(v_{j}-u_{j}\right)\right)\right| \leq C$ a.e. and for every $j \geq 1$ by assumption (f1), for all $j \geq 1$ we find $\xi_{j} \in(0,1)$ such that from formula (5.8) we obtain

$$
\begin{aligned}
I^{\prime}\left(v_{j}\right)(\varphi)-I^{\prime}\left(u_{j}\right)(\varphi) & =I^{\prime \prime}\left(u_{j}+\xi_{j}\left(v_{j}-u_{j}\right)\right)\left(\varphi, v_{j}-u_{j}\right) \\
& =\int(-\Delta)^{s / 2} \varphi(-\Delta)^{s / 2}\left(v_{j}-u_{j}\right)+\lambda \int \varphi\left(v_{j}-u_{j}\right) \\
& -\int a(x) f^{\prime}\left(u_{j}+\xi_{j}\left(v_{j}-u_{j}\right)\right) \varphi\left(v_{j}-u_{j}\right) \\
& \leq C\|\varphi\|_{H^{s}}\left\|v_{j}-u_{j}\right\|_{H^{s}}+C a_{\infty} \int\left|\varphi\left\|v_{j}-u_{j} \mid \leq C\right\| \varphi\left\|_{H^{s}}\right\| v_{j}-u_{j} \|_{H^{s}} .\right.
\end{aligned}
$$

In turn, taking the supremum over the $\varphi \in H^{s}\left(\mathbb{R}^{n}\right)$ with $\|\varphi\|_{H^{s}} \leq 1$, we get as $j \rightarrow \infty$

$$
\left\|I^{\prime}\left(v_{j}\right)-I^{\prime}\left(u_{j}\right)\right\|_{H^{-s}} \leq C\left\|v_{j}-u_{j}\right\|_{H^{s}}=o_{j}(1)
$$

which concludes the proof.

Now we define

$$
b:=\inf \{I(u): u \in \mathcal{P} \text { and } \beta(u)=0\} .
$$

It is clear that $b \geq c_{\infty}$. Moreover, we have the following

Lemma 5.13. $b>c_{\infty}$.

Proof. Suppose $b=c_{\infty}$. By definition, there exists a sequence $\left\{u_{j}\right\}$ with $u_{j} \in \mathcal{P}$ and $\beta\left(u_{j}\right)=0$ such that $I\left(u_{j}\right) \rightarrow b$. By Lemma 5.10, $\left\{u_{j}\right\}$ is bounded. Since $b=p$ by Lemmas 5.2 and 5.3, then $\left\{u_{j}\right\}$ is also a minimizing sequence of $I$ on $\mathcal{P}$. By Ekeland Variational Principle, there exists another sequence $\left\{\tilde{u}_{j}\right\} \subset \mathcal{P}$ such that $I\left(\tilde{u}_{j}\right) \rightarrow p,\left.I^{\prime}\right|_{\mathcal{P}}\left(\tilde{u}_{j}\right) \rightarrow 0$ and $\left\|\tilde{u}_{j}-u_{j}\right\|_{H^{s}} \rightarrow 0$ as $j \rightarrow \infty$. Let us now prove that $I^{\prime}\left(\tilde{u}_{j}\right) \rightarrow 0$, as $j \rightarrow \infty$. Suppose by contradiction that this is not the case. Then, there exists $\sigma>0$ and a subsequence $\left\{\tilde{u}_{j_{k}}\right\}$ with

$$
\left\|I^{\prime}\left(\tilde{u}_{j_{k}}\right)\right\|>\sigma, \quad \text { for all } k \geq 1 \text { large. }
$$

Arguing as in the proof of Lemma 5.12, there exists a positive constant $C$ such that

$$
\left|I^{\prime}\left(\tilde{u}_{j_{k}}\right)(\varphi)-I^{\prime}(v)(\varphi)\right| \leq C\left\|\tilde{u}_{j_{k}}-v\right\|_{H^{s}}\|\varphi\|_{H^{s}}, \quad \text { for all } k \geq 1 \text { and any } v, \varphi \in H^{s}\left(\mathbb{R}^{n}\right) .
$$

Taking the supremum over $\|\varphi\|_{H^{s}} \leq 1$ yields $\left\|I^{\prime}\left(\tilde{u}_{j_{k}}\right)-I^{\prime}(v)\right\|_{H^{-s}} \leq C\left\|\tilde{u}_{j_{k}}-v\right\|_{H^{s}}$ for all $k \geq 1$ and any $v \in H^{s}\left(\mathbb{R}^{n}\right)$. Therefore, if $\left\|\tilde{u}_{j_{k}}-v\right\|_{H^{s}}<\tilde{\delta} / C:=2 \delta$, then we have $\left\|I^{\prime}\left(\tilde{u}_{j_{k}}\right)-I^{\prime}(v)\right\|_{H^{-s}}<\tilde{\delta}$. for all $v \in H^{s}\left(\mathbb{R}^{n}\right)$ and $k \geq 1$. This yields, $\sigma-\tilde{\delta}<\left\|I^{\prime}\left(\tilde{u}_{j_{k}}\right)\right\|_{H^{-s}}-\tilde{\delta}<\left\|I^{\prime}(v)\right\|_{H^{-s}}$, for all $k \geq 1$ large. For $\tilde{\delta} \in(0, \sigma)$, we have $\lambda:=\sigma-\tilde{\delta}>0$ and

$$
\forall v \in H^{s}\left(\mathbb{R}^{n}\right): \quad v \in B_{2 \delta}\left(\tilde{u}_{j_{k}}\right) \Longrightarrow\left\|I^{\prime}(v)\right\|_{H^{-s}}>\lambda .
$$

Let us now set $\varepsilon:=\min \{p / 2, \lambda \delta / 8\}$ and $S:=\left\{\tilde{u}_{j_{k}}\right\}$. Then, by virtue of [30, Lemma 2.3], there is a deformation $\eta:[0,1] \times H^{s}\left(\mathbb{R}^{n}\right) \rightarrow H^{s}\left(\mathbb{R}^{n}\right)$ at the level $p$, such that

$$
\eta\left(1, I^{p+\varepsilon} \cap S\right) \subset I^{p-\varepsilon}, \quad I(\eta(1, u)) \leq I(u), \quad \text { for all } u \in H^{s}\left(\mathbb{R}^{n}\right) .
$$

For $k$ large enough, since $\tilde{u}_{j_{k}}$ is minimizing for $p$, we have

$$
\max _{t>0} I\left(\tilde{u}_{j_{k}}(\cdot / t)\right)=I\left(\tilde{u}_{j_{k}}\right)<p+\varepsilon .
$$

Observe that, for each $k \geq 1$, by $(A 4)$ we have

$$
\int G_{\infty}\left(\tilde{u}_{j_{k}}\right) \geq \int\left(\left(a(x)+\frac{\nabla a(x) \cdot x}{n}\right) F\left(\tilde{u}_{j_{k}}\right)-\lambda \frac{\tilde{u}_{j_{k}}^{2}}{2}\right)=\frac{n-2 s}{2 n} \int\left|(-\Delta)^{s / 2} \tilde{u}_{j_{k}}\right|^{2}>0
$$


so that the arguments of Lemma 4.1 work for $\tilde{u}_{j_{k}}$. Since $\tilde{u}_{j_{k}} \in \mathcal{P}$, the first equality in (5.10) is justified by means of formula (4.2) of Lemma 4.1 on $\Psi^{\prime}$, by the uniqueness of positive zeros of $\Psi^{\prime}$ and since $\Psi(\vartheta)>0$ for $\vartheta$ small and $\Psi(\vartheta)<0$ for $\vartheta$ large. Then, we can infer that

$$
\max _{t>0} I\left(\eta\left(1, \tilde{u}_{j_{k}}(\cdot / t)\right)<p-\varepsilon .\right.
$$

On the other hand, for $k$ and $L$ fixed large, $\gamma(t):=\eta\left(1, \tilde{u}_{j_{k}}(\cdot / L t)\right)$ is a path in $\Gamma$ since by (4.1)

$$
\begin{aligned}
I(\gamma(1)) & =I\left(\eta\left(1, \tilde{u}_{j_{k}}(\cdot / L)\right)\right) \leq I\left(\tilde{u}_{j_{k}}(\cdot / L)\right)=\frac{L^{n-2 s}}{2} \int\left|(-\Delta)^{s / 2} \tilde{u}_{j_{k}}\right|^{2}-L^{n} \int\left(a(L x) F\left(\tilde{u}_{j_{k}}\right)-\lambda \frac{\tilde{u}_{j_{k}}^{2}}{2}\right) \\
& =\frac{L^{n-2 s}}{2} \int\left|(-\Delta)^{s / 2} \tilde{u}_{j_{k}}\right|^{2}-L^{n}\left(\int G_{\infty}\left(\tilde{u}_{j_{k}}\right)+o_{L}(1)\right)<0, \quad \text { for } L \rightarrow \infty .
\end{aligned}
$$

Hence, we deduce that

$$
c \leq \max _{t \in[0,1]} I\left(\eta\left(1, \tilde{u}_{j_{k}}(\cdot / L t)\right)=\max _{t>0} I\left(\eta\left(1, \tilde{u}_{j_{k}}(\cdot / t)\right)<p-\varepsilon<p,\right.\right.
$$

contradicting that fact that $p=c$, provided by Lemma 5.3. By Lemma 5.12, being $\left\|\tilde{u}_{j}-u_{j}\right\|_{H^{s}} \rightarrow 0$, we get $I^{\prime}\left(u_{j}\right) \rightarrow 0$ as $j \rightarrow \infty$. Therefore, $\left\{u_{j}\right\}$ satisfies the assumptions of Corollary 5.8 and since $p=c_{\infty}$ is not attained by Theorem 1.1, then the splitting lemma holds with $k=1$, see Corollary 5.8. This yields $u_{j}(x)=u^{1}\left(x-y_{j}\right)+o_{j}(1)$ as $j \rightarrow \infty$ where $y_{j} \in \mathbb{R}^{n},\left|y_{j}\right| \rightarrow+\infty$ and $u^{1}$ is a solution of the problem at infinity. By making a translation, $u_{j}\left(x+y_{j}\right)=u^{1}(x)+o_{j}(1)$. Applying the barycenter map yields $\beta\left(u_{j}\left(x+y_{j}\right)\right)=\beta\left(u_{j}\right)-y_{j}=-y_{j}$ and $\beta\left(u^{1}(x)+o_{j}(1)\right)=\beta\left(u^{1}(x)\right)+o_{j}(1)$ by continuity. Then, we reach a contradiction, yielding $b>c_{\infty}$.

Let us consider a positive, radially symmetric, ground state solution $w \in H^{s}\left(\mathbb{R}^{n}\right)$ to the autonomous problem at infinity. We define the operator $\Pi: \mathbb{R}^{n} \rightarrow \mathcal{P}$ by

$$
\Pi[y](x):=w\left(\frac{x-y}{\vartheta_{y}}\right),
$$

where $\vartheta_{y}$ projects $w(\cdot-y)$ onto $\mathcal{P}$. $\Pi$ is continuous as $\vartheta_{y}$ is unique and $\vartheta_{y}(w(\cdot-y))$ is a continuous function of $w(\cdot-y)$.

Lemma 5.14. $\beta(\Pi[y](x))=y$ for every $y \in \mathbb{R}^{n}$.

Proof. Let $v(x)=w\left((x-y) / \vartheta_{y}\right)$, then

$$
\mu(v)(x)=\frac{1}{\left|B_{1}\right|} \int_{B_{1}(x-y)}\left|w\left(\frac{\xi}{\vartheta_{y}}\right)\right| d \xi=\mu\left(w\left(\frac{\cdot}{\vartheta_{y}}\right)\right)(x-y),
$$

and further, that $\left.\hat{v}(x)=\widehat{w\left(\cdot / \vartheta_{y}\right.}\right)(x-y)$. Using the fact that $\|\hat{v}\|_{L^{1}}=\left\|\widehat{w\left(\cdot / \vartheta_{y}\right)}\right\|_{L^{1}}$, we get

$$
\begin{aligned}
\beta(v) & \left.=\frac{1}{\|\hat{v}\|_{L^{1}}} \int x \widehat{w\left(\cdot / \vartheta_{y}\right.}\right)(x-y) d x \\
& \left.=\frac{1}{\|\hat{v}\|_{L^{1}}} \int(z+y) \widehat{w\left(\cdot / \vartheta_{y}\right.}\right)(z) d z \\
& \left.\left.=\frac{1}{\|\hat{v}\|_{L^{1}}} \int z \widehat{w\left(\cdot / \vartheta_{y}\right.}\right)(z) d z+\frac{1}{\|\hat{v}\|_{L^{1}}} \int y \widehat{w\left(\cdot / \vartheta_{y}\right.}\right)(z) d z \\
& =\beta\left(w\left(\cdot / \vartheta_{y}\right)\right)+\frac{y}{\|\hat{v}\|_{L^{1}}} \int \hat{v}(y+z) d z=y,
\end{aligned}
$$

since $w$ is radially symmetric.

Lemma 5.15. $I(\Pi[y]) \searrow c_{\infty}$, if $|y| \rightarrow+\infty$. 
Proof. Since $\Pi[y] \in \mathcal{P}$, as observed in (4.6), the functional $I$ can be written as

$$
I(\Pi[y])=\frac{s}{n} \int\left|(-\Delta)^{s / 2} w\left(\frac{x-y}{\vartheta_{y}}\right)\right|^{2}+\frac{1}{n} \int \nabla a(x) \cdot x F\left(w\left(\frac{x-y}{\vartheta_{y}}\right)\right) .
$$

Moreover, since $w \in \mathcal{P}_{\infty}$, by (4.7) we have $I_{\infty}(w)=\frac{s}{n} \int\left|(-\Delta)^{s / 2} w\right|^{2}$ and we obtain

$$
\begin{aligned}
I(\Pi[y]) & =\frac{s \vartheta_{y}^{n-2 s}}{n} \int\left|(-\Delta)^{s / 2} w\right|^{2} \\
& +\frac{\vartheta_{y}^{n}}{n} \int \nabla a\left(\vartheta_{y} x+y\right) \cdot\left(\vartheta_{y} x+y\right) F(w) \\
& =\vartheta_{y}^{n-2 s} I_{\infty}(w)+\frac{\vartheta_{y}^{n}}{n} \int \nabla a\left(\vartheta_{y} x+y\right) \cdot\left(\vartheta_{y} x+y\right) F(w) \quad\left(>c_{\infty}\right) .
\end{aligned}
$$

By Lebesgue Dominated Convergence Theorem, (1.7) and $\vartheta_{y} \rightarrow 1$ if $|y| \rightarrow+\infty$, we get

$$
\lim _{|y| \rightarrow \infty} \int \nabla a\left(\vartheta_{y} x+y\right) \cdot\left(\vartheta_{y} x+y\right) F(w)=0 .
$$

Therefore, $I(\Pi[y]) \searrow c_{\infty}$ if $|y| \rightarrow+\infty$ and the proof is complete.

Lemma 5.16. Let $C$ be a positive constant such that $|F(s)| \leq C s^{2}$. Assume

$$
(A 6)\left\|a_{\infty}-a\right\|_{L^{\infty}}<\frac{\min \left\{c_{\sharp}, 2 c_{\infty}\right\}-c_{\infty}}{\widehat{\vartheta}^{n}\|w\|_{2}^{2} C}, \quad \widehat{\vartheta}=\sup _{y \in \mathbb{R}^{n}} \vartheta_{y} .
$$

Then $I(\Pi[y])<\min \left\{c_{\sharp}, 2 c_{\infty}\right\}$ for every $y \in \mathbb{R}^{n}$.

Proof. The maximum of $t \mapsto I_{\infty}(w(\cdot / t))$ is attained at $t=1$. Since $\vartheta_{y}>1$, using $(A 6)$, we obtain

$$
\begin{aligned}
I(\Pi[y]) & =I_{\infty}(\Pi[y])+I(\Pi[y])-I_{\infty}(\Pi[y]) \leq I_{\infty}(w)+\int\left(a_{\infty}-a(x)\right) F(\Pi[y]) \\
& <c_{\infty}+\frac{\min \left\{c_{\sharp}, 2 c_{\infty}\right\}-c_{\infty}}{\widehat{\vartheta}^{n}\|w\|_{2}^{2} C} \int C w^{2}\left(\frac{x-y}{\vartheta_{y}}\right) \\
& =c_{\infty}+\frac{\left(\min \left\{c_{\sharp}, 2 c_{\infty}\right\}-c_{\infty}\right) \vartheta_{y}^{n}}{\widehat{\vartheta}^{n}\|w\|_{2}^{2}}\|w\|_{2}^{2}=\min \left\{c_{\sharp}, 2 c_{\infty}\right\},
\end{aligned}
$$

which concludes the proof.

Remark 5.17. Replacing (A6) with $\left\|a_{\infty}-a\right\|_{L^{\infty}}<c_{\infty} \widehat{\vartheta}^{-n}\|w\|_{2}^{-2} C^{-1}$, one gets $I(\Pi[y])<2 c_{\infty}$.

We will need a version of the Linking Theorem with Cerami condition by [4, Theorem 2.3].

Definition 5.18. Let $S$ be a closed subset of a Banach space $X$ and $Q$ a sub manifold of $X$ with relative boundary $\partial Q$. We say that $S$ and $\partial Q$ link if the following facts hold

1) $S \cap \partial Q=\emptyset$;

2) for any $h \in C^{0}(X, X)$ with $\left.h\right|_{\partial Q}=i d$, then $h(Q) \cap S \neq \emptyset$.

Moreover, if $S$ and $Q$ are as above and $B$ is a subset of $C^{0}(X, X)$, then $S$ and $\partial Q$ link with respect to $B$ if 1) and 2) hold for any $h \in B$.

Theorem 5.19. Suppose that $I \in C^{1}(X, \mathbb{R})$ is a functional satisfying $(C e)$ condition. Consider a closed subset $S \subset X$ and a submanifold $Q \subset X$ with relative boundary $\partial Q$ such that

a) $S$ and $\partial Q$ link;

b) $\alpha=\inf _{u \in S} I(u)>\sup _{u \in \partial Q} I(u)=\alpha_{0}$.

c) $\sup _{u \in Q} I(u)<+\infty$. 
If $B=\left\{h \in C^{0}(X, X):\left.h\right|_{\partial Q}=\mathrm{id}\right\}$, then $\tau=\inf _{h \in B} \sup _{u \in Q} I(h(u)) \geq \alpha$ is a critical value of $I$.

Proof of Theorem 1.2 concluded. We follow the argument in [1, Theorem 7.7]. Since we have $b>c_{\infty}$ from Lemma 5.13 and $I(\Pi[y]) \searrow c_{\infty}$ if $|y| \rightarrow \infty$ from Lemma 5.15, there exists $\bar{\rho}>0$ such that

$$
c_{\infty}<\max _{|y|=\bar{\rho}} I(\Pi[y])<b .
$$

In order to apply the linking theorem, we take

$$
Q:=\Pi\left(\overline{B_{\bar{\rho}}(0)}\right), \quad S:=\left\{u \in H^{s}\left(\mathbb{R}^{n}\right): u \in \mathcal{P}, \beta(u)=0\right\}
$$

and we show that $\partial Q$ and $S$ link with respect to $\mathcal{H}=\left\{h \in C(Q, \mathcal{P}):\left.h\right|_{\partial Q}=i d\right\}$. Since $\beta(\Pi[y])=y$ from Lemma 5.14, we have that $\partial Q \cap S=\emptyset$, as if $u \in S$, then $\beta(u)=0$, and if $u \in \partial Q, u=\Pi[y]$ for some $y \in \mathbb{R}^{n}$ with $|y|=\bar{\rho}$ and then $\beta(u)=y \neq 0$. Now we show that $h(Q) \cap S \neq \emptyset$ for any $h \in \mathcal{H}$. Given $h \in \mathcal{H}$, let $T: \overline{B_{\bar{\rho}}(0)} \rightarrow \mathbb{R}^{n}$ by defined by $T(y)=\beta \circ h \circ \Pi[y]$. The function $T$ is continuous, by composition. Moreover, for $|y|=\bar{\rho}$, we have that $\Pi[y] \in \partial Q$, thus $h \circ \Pi[y]=\Pi[y]$, as $\left.h\right|_{\partial Q}=i d$, and hence $T(y)=y$ by Lemma 5.14. By Brower Fixed Point Theorem there is $\tilde{y} \in B_{\bar{\rho}}(0)$ with $T(\tilde{y})=0$, which implies $h(\Pi[\tilde{y}]) \in S$. Then $h(Q) \cap S \neq \emptyset$ and $S$ and $\partial Q$ link. Now, from (5.11), we may write

Let us define

$$
b=\inf _{S} I>\max _{\partial Q} I
$$

$$
d=\inf _{h \in \mathcal{H}} \max _{u \in Q} I(h(u)) .
$$

It is $d \geq b$. In fact, if $h \in \mathcal{H}$, there exists $w \in S$ with $w=h(v)$ for some $v \in \Pi\left(\overline{B_{\bar{\rho}}(0)}\right)$. Therefore,

$$
\max _{u \in Q} I(h(u)) \geq I(h(v))=I(w) \geq \inf _{u \in S} I(u)=b,
$$

and hence $d \geq b$, which implies $d>c_{\infty}$. Furthermore, if $h=i d$, then

$$
\inf _{h \in \mathcal{H}} \max _{u \in Q} I(h(u))<\max _{u \in Q} I(u)<\min \left\{c_{\sharp}, 2 c_{\infty}\right\},
$$

in light of Lemma 5.16. Then $d \in\left(c_{\infty}, \min \left\{c_{\sharp}, 2 c_{\infty}\right\}\right)$ and thus from Lemma 5.9 the $(C e)$ condition is satisfied at level $d$. Then, by the linking theorem, $d$ is a critical level for $I$.

\section{REFERENCES}

[1] A. Ambrosetti, G. Cerami, D. Ruiz, Solitons of linearly coupled systems of semilinear non-autonomous equations on $\mathbb{R}^{n}$, J. Funct. Anal. 254 (2008), 2816-2845. 15, 23

[2] A. Ambrosetti and P.H. Rabinowitz, Dual variational methods in critical point theory and applications, J. Funct. Anal. 14, (1973), 349-381. 5

[3] A. Azzollini, A. Pomponio, On the Schrödinger equation in $\mathbb{R}^{n}$ under the effect of a general nonlinear term, Indiana Univ. Math. J. 58 (2009), 1361-1378. 3

[4] P. Bartolo, V. Benci, D. Fortunato, Abstract critical point theorems and applications to some nonlinear problems with strong resonance at infinity, Nonlinear Anal. 7 (1983), 981-1012. 22

[5] J. Byeon, L. Jeanjean, M. Maris, Symmetry and monotonicity of least energy solutions, Calc. Var. Partial Differential Equations 36 (2009), 481-492. 2, 6

[6] L. Caffarelli, L. Silvestre, An extension problem related to the fractional Laplacian, Comm. Partial Differential Equations 32 (2007) 1245-1260. 1

[7] X. Chang, Ground state solutions of asymptotically linear fractional Schr Ãúdinger equations, J. Math. Phys $\mathbf{5 4}$ (2013) 061504. 3

[8] X. Chang, Z-Q. Wang, Ground state of scalar field equations involving a fractional Laplacian with general nonlinearity, Nonlinearity 26 (2013), 479-494. 3, 4, 5

[9] D.G. Costa, H. Tehrani, On a class of asymptotically linear elliptic problems in $\mathbb{R}^{n}$, J. Differential Equations 173, (2001), 470-494. 3 
[10] E. Di Nezza, G. Palatucci, E. Valdinoci, Hitchhiker's guide to the fractional Sobolev spaces, Bull. Sci. Math. 136 (2012), 512-573. 1, 6, 7, 12

[11] I. Ekeland, Convexity methods in Hamiltonian mechanics, Springer-Verlag in Berlin, New York, (1990). 17

[12] P. Felmer, A. Quaas, J. Tan, Positive solutions of nonlinear Schrödinger equation with the fractional Laplacian, Proc. Royal Soc. Edinburgh A 142 (2012), 1237-1262. 3, 18

[13] R.L. Frank, E. Lenzmann, Uniqueness and non degeneracy of ground states for $(-\Delta)^{s} Q+Q-Q^{\alpha+1}=0$ in $\mathbb{R}$, Acta Math. 210 (2013), 261-318. 3

[14] R.L. Frank, E. Lenzmann, L. Silvestre, Uniqueness of radial solutions for the fractional laplacian, preprint 3

[15] N. Ghoussoub, D. Preiss, A general mountain pass principle for locating and classifying critical points, Ann. Inst. Henri Poincarè Analyse Non Linèaire 6 (1989), 321-330. 17

[16] L. Jeanjean, K. Tanaka, A remark on least energy solutions in $\mathbb{R}^{N}$, Proc. Amer. Math. Soc. 131 (2002), 23992408. 3,4

[17] N. Laskin, Fractional Schrödinger equations, Phys. Rev. E 66 (2002), 056108. 1

[18] R. Lehrer, L.A. Maia, Positive solutions to asymptotically linear equations via Pohǒzaev manifold, J. Funct. Anal 266 (2014), 213-246. 3

[19] P L. Lions, The concentration-compactness principle in the calculus of variations. The locally compact case, Ann. Inst. Henri Poincarè Analyse Non Linèaire 1 (1984), 109-145 and 223-283. 16

[20] O. Lopes, M. Maris, Symmetry of minimizers for some nonlocal variational problems, J. Funct. Anal. 254 (2008), 535-592. 2

[21] R. Metzler, J. Klafter, The random walkâĂ Źs guide to anomalous diffusion: a fractional dynamics approach, Phys. Rep. 339 (2000), 1-77. 1

[22] R. Metzler, J. Klafter, The restaurant at the random walk: recent developments in the description of anomalous transport by fractional dynamics, J. Phys. A 37 (2004), 161-208. 1

[23] X. Ros-Oton, J. Serra, The Pohozaev identity for the fractional laplacian, preprint. 4, 7

[24] J. Serrin, M. Tang, Uniqueness of ground states for quasilinear elliptic equations, Indiana Univ. J. Math 49 (2000), 897-923. 3

[25] G.S. Spradlin, Existence of solutions to a hamiltonian System without convexity condition on the nonlinearity, Electron. J. Differential Equations 21 (2004), 1-13. 15

[26] G.S. Spradlin, Interacting near-solutions of a hamiltonian system, Calc. Var. Partial Differential Equations 22 (2005), 447-464. 15

[27] C.A. Stuart, H.S. Zhou, Applying the mountain pass theorem to an asymptotically linear elliptic equation on $\mathbb{R}^{n}$, Comm. Partial Differential Equations 9-10 (1999), 1731-1758. 3

[28] C. A. Stuart, Guidance properties of nonlinear planar waveguides, Arch. Rational Mech. Anal. 125 (1993), 145-200. 3

[29] K. Tintarev, K.H. Fieseler, Concentration compactness, Imperial College Press, London, vi +264.18

[30] M. Willem, Minimax Theorems, 24 Birkhauser, Boston, 1996. 20

\author{
Centro de Ciencias Exatas e Tecnologicas \\ CCET, Unioeste \\ CASCAVEL-PR, BRAZIL \\ E-mail address: rlehrer@gmail.com \\ Departamento de Matematica \\ UNIVERSIDADE DE BRASILIA \\ Brasilia, BrazIL \\ E-mail address: lilimaia.unb@gmail.com \\ Dipartimento Di InFORMATICA \\ Università degli Studi di Verona \\ VERONA, ITALY \\ E-mail address: marco.squassina@univr.it
}

